\title{
Embedding codimension of the space of arcs
}

\author{
Christopher Chiu ${ }^{1,2}$, Tommaso de Fernex ${ }^{3}$ and Roi Docampo 4 \\ ${ }^{1}$ Fakultät für Mathematik, Universität Wien, Oskar-Morgenstern-Platz 1, A-1090 Vienna, Austria. \\ ${ }^{2}$ Department of Mathematics and Computer Science, Eindhoven University of Technology, De Groene Loper 5, 5612 AZ \\ Eindhoven, the Netherlands; E-mail: c.h.chiu@tue.nl. \\ ${ }^{3}$ Department of Mathematics, University of Utah, 155 South 1400 East, Salt Lake City, UT 84112, USA; \\ E-mail: defernex@math.utah.edu. \\ ${ }^{4}$ Department of Mathematics, University of Oklahoma, 601 Elm Ave., Room 423, Norman, OK 73019, USA; \\ E-mail: roi@ou.edu.
}

Received: 23 January 2020; Accepted: 15 December 2021

2020 Mathematics Subject Classification: Primary - 14E18, 13B35; Secondary - 14B05, 14B20, 13C15, 13F25

\begin{abstract}
We introduce a notion of embedding codimension of an arbitrary local ring, establish some general properties and study in detail the case of arc spaces of schemes of finite type over a field. Viewing the embedding codimension as a measure of singularities, our main result can be interpreted as saying that the singularities of the arc space are maximal at the arcs that are fully embedded in the singular locus of the underlying scheme, and progressively improve as we move away from said locus. As an application, we complement a theorem of Drinfeld, Grinberg and Kazhdan on formal neighbourhoods in arc spaces by providing a converse to their theorem, an optimal bound for the embedding codimension of the formal model appearing in the statement, a precise formula for the embedding dimension of the model constructed in Drinfeld's proof and a geometric meaningful way of realising the decomposition stated in the theorem.
\end{abstract}

\section{Contents}

1 Introduction $\quad 1$

2 Rings of formal power series $\quad \mathbf{5}$

3 Embedding dimension $\quad \mathbf{6}$

4 Flatness of completion $\quad 10$

5 Ideals of finite definition $\quad 13$

6 Embedding codimension $\quad 17$

7 Formal embedding codimension $\quad 19$

8 Embedding codimension of arc spaces $\quad \mathbf{2 2}$

9 On Drinfeld, Grinberg and Kazhdan's theorem 28

10 Efficient embedding of the Drinfeld model 31

11 Applications to Mather-Jacobian discrepancies $\quad 34$

\section{Introduction}

In this paper we define the embedding codimension of an arbitrary local ring and use it to quantify singularities of arc spaces.

(C) The Author(s), 2022. Published by Cambridge University Press. This is an Open Access article, distributed under the terms of the Creative Commons Attribution licence (https://creativecommons.org/licenses/by/4.0/), which permits unrestricted re-use, distribution, and reproduction in any medium, provided the original work is properly cited. 
The embedding codimension is a familiar notion in the Noetherian setting, where it is defined, for local rings, as the difference between the embedding dimension and the Krull dimension. It was studied, for instance, in [35] under the name of regularity defect. Note, however, that if the ring is not Noetherian, then both of these quantities can be infinite, and even when they are finite it can happen that the embedding dimension is smaller than the dimension. Rank 2 valuation rings give simple examples where this phenomenon occurs.

Arc spaces provide a situation of geometric interest where non-Noetherian rings and rings of infinite embedding dimension naturally arise. With this in mind, we extend the definition of the embedding codimension to arbitrary local rings $(A, \mathfrak{m}, k)$ by setting

$$
\operatorname{ecodim}(A):=\operatorname{ht}(\operatorname{ker}(\gamma))
$$

where $\gamma: \operatorname{Sym}_{k}\left(\mathrm{~m} / \mathrm{m}^{2}\right) \rightarrow \operatorname{gr}(A)$ is the natural homomorphism. Geometrically, we may think of $\operatorname{ecodim}(A)$ as the codimension of the tangent cone of $A$ inside its tangent space. Note that when $A$ is Noetherian, this notion agrees with the classical definition of the embedding codimension, as in this case $\operatorname{dim}(A)=\operatorname{dim}(\operatorname{gr}(A))$. When $A$ is a $k$-algebra, we have ecodim $(A)=0$ if and only if $A$ is formally smooth over $k$, and therefore one can view the embedding codimension as a (rough) measure of singularity.

If $(A, \mathfrak{m}, k)$ is equicharacteristic, then an alternative definition can be given by considering the infimum of $h \mathrm{t}(\operatorname{ker}(\tau))$ for all surjective continuous $\tau: k\left[\left[x_{i} \mid i \in I\right]\right] \rightarrow \widehat{A}$. We call the resulting notion the formal embedding codimension, and denote it by $\operatorname{fcodim}(A)$. In this paper we establish the following comparison theorem:

Theorem A. For every equicharacteristic local ring $(A, \mathrm{~m}, k)$, we have

$$
\operatorname{ecodim}(A) \leq \operatorname{fcodim}(A)
$$

and equality holds in the following cases:

1. the ring $A$ has embedding dimension $\operatorname{edim}(A)<\infty$ or

2. there exists a scheme $X$ of finite type over $k$ such that $A$ is isomorphic to the local ring of the arc space of $X$ at a $k$-rational point.

In order to prove Theorem A and related results on the formal embedding codimension, we make use of various results concerning infinite-variate power series rings and their localisations, which are of independent interest in the study of non-Noetherian rings.

Local rings of arc spaces and their completions were studied in [31, 24, 40, 41, 42, 37, 21]; [21] looks at the embedding dimension of the local rings to characterise stable points of arc spaces, which were originally studied in $[23,40]$. In this paper, we consider the embedding codimension.

Let $X$ be a scheme of finite type over a field $k$, and let $X_{\infty}$ be its arc space. A point $\alpha \in X_{\infty}$ corresponds to a formal arc $\alpha: \operatorname{Spec} K[[t]] \rightarrow X$, where $K$ is the residue field, and defines a valuation ord ${ }_{\alpha}$ on the local ring of $X$ at the base point $\alpha(0)$ of the arc (the image of the closed point of $\operatorname{Spec} K[[t]]$ ). It is convenient to denote by $\alpha(\eta)$ the image of the generic point of Spec $K[[t]]$. With this notation, we can state our next theorem.

Theorem B. Let $X$ be a scheme of finite type over a field $k$, and let $\alpha \in X_{\infty}$.

1. Assume that either $k$ has characteristic 0 or $\alpha \in X_{\infty}(k)$. Then we have that $\operatorname{ecodim}\left(\mathcal{O}_{X_{\infty}, \alpha}\right)<\infty$ if and only if $\alpha(\eta) \in X_{\mathrm{sm}}$.

2. Assume that $k$ is perfect and $\alpha(\eta) \in X_{\mathrm{sm}}$, and let $X^{0} \subset X$ be the irreducible component containing $\alpha(\eta)$. Then

$$
\operatorname{ecodim}\left(\mathcal{O}_{X_{\infty}, \alpha}\right) \leq \operatorname{ord}_{\alpha}\left(\operatorname{Jac}_{X^{0}}\right)
$$

where $\mathrm{Jac}_{X^{0}}$ is the Jacobian ideal of $X^{0}$. 
One of the motivations behind this result comes from the following theorem, originally conjectured by Drinfeld and proved by Grinberg and Kazhdan in characteristic 0 and then by Drinfeld in arbitrary characteristic. Here and in the following, we exclude the trivial case where $X$ is, locally, just a reduced point.

Theorem 1.1 ([31, 24, 25]). Let $X$ be a scheme of finite type over a field $k$, and let $\alpha \in X_{\infty}(k)$ be a $k$-rational point. If $\alpha(\eta) \in X_{\mathrm{sm}}$, then there exists a decomposition

$$
\widehat{X_{\infty, \alpha}} \simeq \widehat{Z_{z}} \hat{\times} \Delta^{\mathbb{N}}
$$

where $\widehat{Z_{z}}$ is the formal completion of a scheme $Z$ of finite type over $k$ at a point $z \in Z(k), \Delta^{\mathbb{N}}=$ $\operatorname{Spf}\left(k\left[\left[x_{i} \mid i \in \mathbb{N}\right]\right]\right)$ and $\hat{x}$ denotes the product in the category of formal $k$-schemes.

Given the existence of an isomorphism as in Theorem 1.1, we say that $X_{\infty}$ admits a DGK decomposition at $\alpha$. The germ $(Z, z)$ (given by $\left.\operatorname{Spec} \mathcal{O}_{Z, z}\right)$ and its completion $\widehat{Z_{z}}=\operatorname{Spf}\left(\widehat{\mathcal{O}_{Z_{z}}}\right)$ are often referred to as a formal model for $\alpha$. Drinfeld's proof yields an algorithm for computing such a model; we will refer to it as a Drinfeld model.

Partial converses of Theorem 1.1 were obtained in [9], where an explicit example of a $k$-valued constant arc through the singular locus of $X$ is given for which a DGK decomposition does not exist, and in [19], where it is shown that in characteristic 0 , if $\alpha$ is any constant arc contained in the singular locus of $X$ then there are no smooth factors in $\overline{X_{\infty, \alpha}}$ at all. Examples of nonconstant arcs that are contained in the singular locus for which there is no DGK decomposition can easily be constructed from these results; see also $[43,11]$ for related results. An extension of the theorem to formal schemes is given in [10]. Formal models of toric singularities are studied in [13].

As an application of our methods, we give a sharp converse to Theorem 1.1 and provide an optimal bound to the embedded codimension of the formal model.

Theorem C. Let X be a scheme of finite type over a field $k$, and let $\alpha \in X_{\infty}(k)$ be a k-rational point.

1. If $X_{\infty}$ admits a DGK decomposition at $\alpha$, then $\alpha(\eta) \in X_{\mathrm{sm}}$.

2. Assume that $k$ is perfect and $\alpha(\eta) \in X_{\mathrm{sm}}$, and let $X^{0} \subset X$ be the irreducible component containing $\alpha(\eta)$. Then for any formal model $(Z, z)$ for $\alpha$, we have

$$
\operatorname{ecodim}\left(\mathcal{O}_{Z, z}\right) \leq \operatorname{ord}_{\alpha}\left(\operatorname{Jac}_{X^{0}}\right) .
$$

Moreover, for every $e \in \mathbb{N}$ there exist $X$ and $\alpha$ such that both sides in this formula are equal to $e$.

The next result, which combines results of this paper with Theorem 1.1, provides a geometrically meaningful way of realising a DGK decomposition and gives an explicit formula for the embedding dimension of a Drinfeld model.

Theorem D. Let $X$ be an affine scheme of finite type over a perfect field $k$, let $\alpha \in X_{\infty}(k)$ be a $k$-rational point with $\alpha(\eta) \in X_{\mathrm{sm}}$ and let $d=\operatorname{dim}_{\alpha(\eta)}(X)$. Let $f: X \rightarrow Y:=\mathbb{A}^{d}$ be a general linear projection.

1. The map $f_{\infty}: X_{\infty} \rightarrow Y_{\infty}$ induces an isomorphism from the Zariski tangent space of $X_{\infty}$ at $\alpha$ to the Zariski tangent space of $Y_{\infty}$ at $\beta:=f_{\infty}(\alpha)$, and hence a closed embedding

$$
\widehat{f_{\infty, \alpha}}: \widehat{X_{\infty, \alpha}} \hookrightarrow \widehat{Y_{\infty, \beta}} .
$$

2. For a suitable isomorphism $\widehat{Y_{\infty, \beta}} \simeq \operatorname{Spec} k\left[\left[u_{i} \mid i \in \mathbb{N}\right]\right]$, the scheme $\widehat{X_{\infty, \alpha}}$ is defined in this embedding by finitely many polynomials in the variables $u_{j}$, and hence the embedding gives a DGK decomposition of $X_{\infty}$ at $\alpha$.

3. Let $X^{0} \subset X$ be the irreducible component containing $\alpha(\eta)$, and set $e:=\operatorname{ord}_{\alpha}\left(\operatorname{Jac}_{X^{0}}\right)$. Denote by $\widehat{Y_{2 e-1, \beta_{2 e-1}}}$ the completion of the $(2 e-1)$-jet scheme of $Y$ at the truncation of $\beta$. If $(Z, z)$ is a Drinfeld 
model compatible with the projection $f$, then the composition of maps

$$
\widehat{Z_{z}} \hookrightarrow \widehat{X_{\infty, \alpha}} \hookrightarrow \widehat{Y_{\infty, \beta}} \rightarrow \widehat{Y_{2 e-1, \beta_{2 e-1}}}
$$

gives an embedding of $\widehat{Z_{z}}$ into $\widehat{Y_{2 e-1, \beta_{2 e-1}}}$, and this embedding induces an isomorphism at the level of continuous tangent spaces. In particular, the local ring $\mathcal{O}_{Z, z}$ has embedding dimension

$$
\operatorname{edim}\left(\mathcal{O}_{Z, z}\right)=2 d \operatorname{ord}_{\alpha}\left(\operatorname{Jac}_{X^{0}}\right)
$$

By combining Theorems $\mathrm{C}$ and $\mathrm{D}$, one sees that all Drinfeld models $(Z, z)$ have the same dimension, and this dimension satisfies

$$
(2 d-1) \operatorname{ord}_{\alpha}\left(\operatorname{Jac}_{X^{0}}\right) \leq \operatorname{dim}(Z) \leq 2 d \operatorname{ord}_{\alpha}\left(\operatorname{Jac}_{X^{0}}\right) .
$$

In general, Drinfeld models are different from the minimal formal model. Theorem D implies that $2 d \operatorname{ord}_{\alpha}\left(\mathrm{Jac}_{X^{0}}\right)$ is an upper bound on the embedding dimension of the minimal formal model.

The proofs of Theorems B and D rely on a formula on the sheaf of differentials of $X_{\infty}$ from [21]. A result related to Theorem $\mathrm{D}(1)$, dealing with the case where $f: X \rightarrow Y$ is a generically finite morphism of equidimensional schemes with $X$ smooth, was obtained in [27] using a more direct computation of the map induced at the level of Zariski tangent spaces. General projections to $\mathbb{A}^{d}$ are also used in [24] to set up the proof for the Weierstrass preparation theorem; however, Drinfeld's proof does not lead to

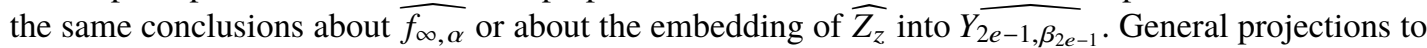
$\mathbb{A}^{d}$ were also used in [42,37], and in fact our results give a new proof of one of the theorems of [37].

There have been several attempts to extend Theorem 1.1 to a more global statement; see [16, 38, 15, 33] (see also the more recent [14], which supersedes [15]), which at their core all rely on the Weierstrass preparation theorem. The question stems from the expectation that there should exist a well-behaved theory of perverse sheaves on arc spaces (as well as on other closely related infinite dimensional spaces). Theorem 1.1 suggests that one could try to define such perverse sheaves in terms of the intersection complexes of the formal models, but one needs a more global version of the DGK decomposition to make sense out of this. We refer to the citations already given for the motivations behind these questions.

Our interest in Theorem $\mathrm{D}$ comes from the observation that the same projection $f: X \rightarrow \mathbb{A}^{d}$ works for all arcs $\alpha^{\prime} \in X_{\infty}(k)$ in a neighbourhood of $\alpha$ and having the same order of contact with $\mathrm{Jac}_{X^{0}}$. The order of contact with the Jacobian ideals of the irreducible components of $X$ gives a stratification of $X_{\infty}$, and the hope is that the theorem may turn out to be useful in understanding how the DGK decomposition varies along strata.

This paper is organised as follows. In the first few sections we review some basic properties of power series rings in an arbitrary number of indeterminates and establish various properties that we have been unable to locate in the literature. Ideals of finite definition, which provide the algebraic interpretation of a DGK decomposition, are discussed in Section 5. These sections provide some general results on non-Noetherian rings and are independent of our applications to the study of singularities of arc spaces. In the next two sections the embedding codimension and its formal counterpart are defined and general properties of these notions are studied. Starting from Section 8, we focus on the case of arc spaces, proving some technical theorems in Section 8 and then addressing the theorem of Drinfeld, Grinberg and Kazhdan in Sections 9 and 10. The last section is devoted to some applications related to MatherJacobian discrepancies, among others to the case of toric singularities using results of [13].

Theorem A follows from Theorem 7.8 and Corollaries 7.5 and 9.7. Theorem B follows from Corollary 8.8 and Theorem 8.5. Theorem C follows from Theorem 9.4 and Example 9.6. Theorem D follows from Theorems 9.8 and 10.2. 


\section{Rings of formal power series}

In this paper we will work with rings of power series in an arbitrary number of indeterminates. For our purposes, we adopt the definition of these rings as completions of polynomial rings, a definition that differs from other standard approaches to the theory. In this section, we briefly review the notions of completions, graded rings, and rings of power series. All of the material here is standard, but we want to fix notation and bring attention to some of the subtleties that appear in the infinite-dimensional setting.

Let $A$ be a ring and $\mathrm{m} \subset A$ an ideal. We do not assume that $\mathrm{m}$ is a maximal ideal. We denote by $\widehat{A}:=\lim _{n} A / \mathrm{m}^{n}$ the $\mathrm{m}$-adic completion of $A$ and regard it as a topological ring with respect to its limit topology. Given an ideal $\mathfrak{a} \subset A$, we denote by $\widehat{\mathfrak{a}} \subset \widehat{A}$ the completion of $\mathfrak{a}$ as a topological $A$-submodule. A basis for all neighbourhoods $U \subset \widehat{A}$ of 0 is given by the descending chain of ideals

$$
\widehat{\mathrm{m}^{n}}=\operatorname{ker}\left(\widehat{A} \rightarrow A / \mathrm{m}^{n}\right)
$$

The ideal $\widehat{\mathfrak{a}}$ then coincides with the topological closure of $\mathfrak{a}$ inside $\widehat{A}-$ that is,

$$
\widehat{\mathfrak{a}}=\bigcap_{n}\left(\mathfrak{a}+\widehat{\mathfrak{m}^{n}}\right) .
$$

Note that if $m$ is not finitely generated, then the natural topology on $\widehat{A}$ may differ from the $\widehat{m}$-adic topology of $\widehat{A}$ (see Remark 2.3).

We will denote by $\operatorname{gr}_{\mathfrak{m}}(A):=\bigoplus_{n \geq 0} \mathrm{~m}^{n} / \mathrm{m}^{n+1}$ the graded algebra of $A$ with respect to the $\mathrm{m}$ adic filtration. If $\mathfrak{m}$ is understood from context, we simply write $\operatorname{gr}(A)$ for $\operatorname{gr}_{\mathfrak{m}}(A)$. We will regard $\widehat{A}$ as endowed with the filtration $\left\{\widehat{\mathrm{m}^{n}}\right\}$ induced by the completion, and therefore its graded algebra is given by $\operatorname{gr}(\widehat{A}):=\bigoplus_{n \geq 0} \widehat{\mathrm{m}^{n}} / \widehat{\mathrm{m}^{n+1}}$. There are natural isomorphisms $\widehat{\mathrm{m}^{p}} / \widehat{\mathrm{m}^{q}} \simeq \mathrm{m}^{p} / \mathrm{m}^{q}$ for all $p<q$. This gives a natural identification between $\operatorname{gr}(\widehat{A})$ and $\operatorname{gr}(A)$. If $\mathfrak{a} \subset A$ is an ideal of $A$, then we write in $(\mathfrak{a}):=\bigoplus_{n \geq 0}\left(\mathfrak{a} \cap \mathfrak{m}^{n}\right) / \mathfrak{m}^{n+1}$ for the corresponding initial ideal. Similarly, for $\mathfrak{a} \subset \widehat{A}$, we set in $(\mathfrak{a}):=\bigoplus_{n \geq 0}\left(\mathfrak{a} \cap \widehat{\mathfrak{m}^{n}}\right) / \widehat{\mathfrak{m}^{n+1}}$. For an element $f \in A$ (or $\left.f \in \widehat{A}\right)$, we write $\operatorname{in}(f) \in \operatorname{gr}(A)$ for the corresponding initial form.

Let $S$ be a ring, and let $\left\{x_{i} \mid i \in I\right\}$ be a collection of indeterminates indexed by an arbitrary set $I$. We consider the polynomial ring $P=S\left[x_{i} \mid i \in I\right]$ and denote by $\widehat{P}=S\left[\left[x_{i} \mid i \in I\right]\right]$ the completion of $P$ with respect to the ideal $\left(x_{i} \mid i \in I\right)$ - that is,

$$
S\left[\left[x_{i} \mid i \in I\right]\right]:=\lim _{n} S\left[x_{i} \mid i \in I\right] /\left(x_{i} \mid i \in I\right)^{n} .
$$

Definition 2.1. We call $S\left[\left[x_{i} \mid i \in I\right]\right]$ the power series ring in the indeterminates $x_{i}$, with $i \in I$, and with coefficients in $S$.

Remark 2.2. The polynomial ring $S\left[x_{i} \mid i \in I\right]$ is always the colimit (that is, the union) of all $S\left[x_{j} \mid j \in J\right]$ with $J \subset I$ finite. On the other hand, if $I$ infinite, then $S\left[\left[x_{i} \mid i \in I\right]\right]$ is not the colimit of all $S\left[\left[x_{j} \mid j \in J\right]\right]$ with $J \subset I$ finite; it is, however, the colimit of all $S\left[\left[x_{j} \mid j \in J\right]\right]$ with $J \subset I$ countable.

Remark 2.3. Denoting $\mathfrak{m}=\left(x_{i} \mid i \in I\right)$, we have the exact sequence

$$
0 \rightarrow \widehat{\mathfrak{m}^{n}} \rightarrow S\left[\left[x_{i} \mid i \in I\right]\right] \rightarrow S\left[x_{i} \mid i \in I\right] / \mathrm{m}^{n} \rightarrow 0 .
$$

If $I$ is infinite then $S\left[\left[x_{i} \mid i \in I\right]\right]$ is not $\widehat{m}$-adically complete - that is, the natural topology on $S\left[\left[x_{i} \mid i \in I\right]\right]$ coming from the completion does not coincide with the $\widehat{m}$-adic one, as for instance the inclusion $\widehat{m}^{2} \subset \widehat{m^{2}}$ is strict in this case [44, Tag 05JA]. 
Remark 2.4. Let us contrast the foregoing definition of $S\left[\left[x_{i} \mid i \in I\right]\right]$ with the ring of formal power series defined in [8, Chapter III, Section 2.11], which we want to briefly recall. For any set $I$ we denote by $\mathbb{N}^{(I)}$ the set of functions $I \rightarrow \mathbb{N}$ that take only finitely many nonzero values. Then $\mathbb{N}^{(I)}$ is a monoid, which we identify with the collection of monomials in the variables $\left\{x_{i} \mid i \in I\right\}$ by writing $x^{\alpha}=\prod_{i \in I, \alpha(i) \neq 0} x_{i}^{\alpha(i)}$ for every $\alpha \in \mathbb{N}^{(I)}$. The $S$-module $S^{\mathbb{N}^{(I)}}$ can be made into an $S$-algebra as follows: writing an element $a=\left(a_{\alpha}\right)_{\alpha \in \mathbb{N}^{(I)}}$ as $a=\sum_{\alpha \in \mathbb{N}^{(I)}} a_{\alpha} x^{\alpha}$, multiplication is defined via formal extension of $x^{\alpha} \cdot x^{\beta}:=x^{\alpha+\beta}$. We call $S^{\mathbb{N}^{(I)}}$ the ring of Bourbaki power series.

Notice that there is a natural inclusion of rings $S\left[\left[x_{i} \mid i \in I\right]\right] \subset S^{\mathbb{N}^{(I)}}$. This inclusion is an equality if $|I|<\infty$, and is a strict inclusion if $|I|=\infty$, as in this case $\sum_{i \in I} x_{i}$ is in $S^{\mathbb{N}^{(I)}}$ but not in $S\left[\left[x_{i} \mid i \in I\right]\right]$.

Remark 2.5. It is often convenient to expand a formal power series in a subset of the indeterminates, but this becomes delicate in the infinite-dimensional case. Let $I$ and $J$ be arbitrary sets, and let $x_{i}$ and $y_{j}$ be indeterminates indexed by $i \in I$ and $j \in J$, respectively. Dropping for short the index sets from the notation, we have the following natural injections:

$$
S\left[\left[x_{i}\right]\right] \otimes_{S} S\left[\left[y_{j}\right]\right] \hookrightarrow S\left[\left[x_{i}\right]\right]\left[\left[y_{j}\right]\right] \hookrightarrow S\left[\left[x_{i}, y_{j}\right]\right] \hookrightarrow\left(S\left[\left[x_{i}\right]\right]\right)^{\mathbb{N}^{(J)}} .
$$

The first inclusion is always strict, and the other two are equalities if and only if $J$ is finite. For example, if $x=x_{i_{0}}$ and $y=y_{i_{0}}$ are two respective indeterminates, then the series $\sum_{n \geq 0} x^{n} y^{n}$ belongs to $S\left[\left[x_{i}\right]\right]\left[\left[y_{j}\right]\right]$ but is not in the image of $S\left[\left[x_{i}\right]\right] \otimes_{S} S\left[\left[y_{j}\right]\right]$, and if $\mathbb{N} \subset J$ and $x=x_{i_{0}}$ is one of the indeterminates, then the series $\sum_{n \geq 1} y_{n} x^{n}$ belongs to $S\left[\left[x_{i}, y_{j}\right]\right]$ but not to $S\left[\left[x_{i}\right]\right]\left[\left[y_{j}\right]\right]$. Notice that Bourbaki power series are better behaved in this respect, as $S^{\mathbb{N}^{(I \sqcup J)}}=\left(S^{\mathbb{N}^{(I)}}\right)^{\mathbb{N}^{(J)}}=\left(S^{\mathbb{N}^{(J)}}\right)^{\mathbb{N}^{(I)}}$.

Remark 2.6. Let $I$ be an arbitrary set, possibly infinite. We have natural identifications

$$
\operatorname{gr}\left(S\left[\left[x_{i} \mid i \in I\right]\right]\right) \simeq \operatorname{gr}\left(S\left[x_{i} \mid i \in I\right]\right) \simeq S\left[x_{i} \mid i \in I\right] .
$$

Any nonzero power series $f \in S\left[\left[x_{i} \mid i \in I\right]\right]$ can be written as $f=\sum_{n=n_{0}}^{\infty} f_{n}$, where $f_{n} \in S\left[x_{i} \mid i \in I\right]$ is homogeneous of degree $n$ and $f_{n_{0}} \neq 0$. Under the given identification, the initial form of $f$ is given by $\operatorname{in}(f)=f_{n_{0}}$. If $\mathfrak{a} \subset S\left[\left[x_{i} \mid i \in I\right]\right]$ is an ideal, then in( $\left.\mathfrak{a}\right)$ gets identified with the ideal of $S\left[x_{i} \mid i \in I\right]$ generated by the initial forms of elements of $\mathfrak{a}$.

Proposition 2.7. Let $P=S\left[x_{i} \mid i \in I\right]$ and $\widehat{P}=S\left[\left[x_{i} \mid i \in I\right]\right]$, where $S$ is a ring and $I$ a set. Let $\mathfrak{a} \subset \widehat{P}$ be an ideal such that $\operatorname{in}(\mathfrak{a}) \subset P$ is finitely generated. Then $\mathfrak{a}$ is finitely generated and is closed in $\widehat{P}$.

Proof. This is proven in [28, Proposition 7.12] for any ring $R$ which is complete with respect to some filtration $\mathfrak{m}_{i}$.

Question 2.8. Does the converse of Proposition 2.7 hold? That is, is the initial ideal in(a) finitely generated for any finitely generated ideal $\mathfrak{a}$ of $\widehat{P}$ ? Alternatively, is any finitely generated $\mathfrak{a}$ already closed inside $\widehat{P}$ ?

\section{Embedding dimension}

In this section we briefly recall the notion of embedding dimension and review some basic properties.

Definition 3.1. The embedding dimension of a local ring $(A, \mathfrak{m}, k)$ is defined to be

$$
\operatorname{edim}(A):=\operatorname{dim}_{k}\left(\mathfrak{m} / \mathfrak{m}^{2}\right)
$$

The $k$-vector space $\mathrm{m} / \mathrm{m}^{2}$ is called the Zariski cotangent space of $A$. 
When the local ring is equicharacteristic, the embedding dimension can equivalently be computed as the dimension of an embedding of the completion in a formal power series ring. Even more, if $A$ is essentially of finite type over an infinite field $k$, then this embedding exists already at a Zariski-local level (see Theorem 3.15). Before we review these facts, it is convenient to introduce some terminology and discuss some general properties.

Definition 3.2. Let $(A, \mathrm{~m}, k)$ be an equicharacteristic local ring. A formal coefficient field of $A$ is a subfield $K \subset \widehat{A}$ that maps isomorphically to $\widehat{A} / \widehat{m}$ via the residue map.

As it is well known, every equicharacteristic local ring $(A, \mathfrak{m}, k)$ admits a formal coefficient field $K \subset \widehat{A}$ (see Remark 3.11).

In order to talk about a well-behaved notion of cotangent map between completions of non-Noetherian rings, we make the following definition:

Definition 3.3. Let $(A, \mathfrak{m}, k)$ be a local ring. The $k$-vector space $\widehat{\mathrm{m}} / \widehat{\mathrm{m}^{2}}$ is called the continuous Zariski cotangent space of $\widehat{A}$. A collection of elements $a_{i} \in \widehat{A}, i \in I$, are called formal coordinates if their images $\bar{a}_{i}$ in $\widehat{\mathrm{m}} / \widehat{\mathrm{m}^{2}}$ form a basis.

Remark 3.4. The continuous Zariski cotangent space $\widehat{\mathrm{m}} / \widehat{\mathrm{m}^{2}}$ of $\widehat{A}$ is naturally isomorphic to the Zariski cotangent space $\mathrm{m} / \mathrm{m}^{2}$ of $A$, but in general it is not the same as the Zariski cotangent space $\widehat{\mathrm{m}} / \widehat{\mathrm{m}}^{2}$ of $\widehat{A}$, as seen in Remark 2.3.

Remark 3.5. If $(A, \mathfrak{m}, k)$ is a local ring admitting a coefficient field, then the continuous cotangent space of $\widehat{A}$ is isomorphic to $\widehat{\Omega}_{A / k} \otimes_{\widehat{A}} k$, where

$$
\widehat{\Omega}_{A / k}:={\underset{n}{\lim }}_{\left(A / \mathfrak{m}^{n}\right) / k}
$$

is defined as in [32, Chapter $0_{\mathrm{IV}}$, Section 20.7].

Definition 3.6. Let $(A, \mathrm{~m}, k)$ be an equicharacteristic local ring. A formal embedding of $A$ is a surjective continuous homomorphism $\tau: \widehat{P} \rightarrow \widehat{A}$, where $\widehat{P}=k\left[\left[x_{i} \mid i \in I\right]\right]$ is a power series ring. A formal embedding $\tau$ is called efficient if the induced map at the level of continuous Zariski cotangent spaces $\widehat{\mathrm{n}} / \widehat{\mathrm{n}^{2}} \rightarrow \widehat{\mathrm{m}} / \widehat{\mathrm{m}^{2}}$ is an isomorphism.

Proposition 3.7. Let $(A, \mathrm{~m}, k)$ be an equicharacteristic local ring. Let $K \subset \widehat{A}$ be a formal coefficient field, and let $a_{i} \in \widehat{A}, i \in I$, be formal coordinates. Then there exists a unique efficient formal embedding $\tau: \widehat{P}=k\left[\left[x_{i} \mid i \in I\right]\right] \rightarrow \widehat{A}$ such that $\tau(k)=K$ and $\tau\left(x_{i}\right)=a_{i}$. Every efficient formal embedding of $A$ is of this form.

Proof. First, note that for every $n \geq 1$ the composition $K \rightarrow \widehat{A} \rightarrow \widehat{A} / \widehat{\mathrm{m}^{n}}$ is injective (since $K$ maps isomorphically to the residue field $k=\widehat{A} / \widehat{\mathrm{m}}$ ) and hence gives an embedding $K \subset A / \mathrm{m}^{n}$ via the natural isomorphism $\widehat{A} / \widehat{\mathfrak{m}^{n}} \simeq A / \mathrm{m}^{n}$. Letting $P=k\left[x_{i} \mid i \in I\right]$ and $\mathfrak{n}=\left(x_{i} \mid i \in I\right) \subset P$, we have compatible homomorphisms $\tau_{n}: P / \mathfrak{n}^{n} \rightarrow A / \mathfrak{m}^{n}$ such that $\tau_{n}(k)=K$ and $\tau_{n}\left(x_{i}\right)=a_{i}+\mathfrak{m}^{n}$. Taking limits, these maps define $\tau$ and determine it uniquely. By construction, $\operatorname{gr}(\tau)$ is surjective, and hence $\tau$ is surjective by [7, Chapter III, Section 2.8, Corollary 2]. Since $\tau$ induces an isomorphism at the level of continuous Zariski cotangent spaces, we see that it is an efficient formal embedding. For the last statement, notice that if $\tau$ is an efficient formal embedding, then clearly $K=\tau(k)$ is a formal coefficient field and $\tau\left(x_{i}\right)$, $i \in I$, are formal coordinates.

The map $\tau$ in Proposition 3.7 can be interpreted as follows. For short, let $P:=\operatorname{Sym}_{k}\left(\mathrm{~m} / \mathrm{m}^{2}\right)$. Every choice of formal coefficient field $K \subset \widehat{A}$ and formal coordinates $a_{i} \in \widehat{A}, i \in I$, determines an embedding $\mathrm{m} / \mathrm{m}^{2} \hookrightarrow \widehat{A}$ as a $K$-vector space, and hence a map $\tau_{0}: P \rightarrow \widehat{A}$. Letting $x_{i}=a_{i}+\mathrm{m}^{2} \in P$, we get a natural identification $P=k\left[x_{i} \mid i \in I\right]$. Then the map $\tau$ is obtained from $\tau_{0}$ by completing the domain $P$. 
Remark 3.8. There is also a natural embedding $\mathrm{m} / \mathrm{m}^{2} \hookrightarrow \operatorname{gr}(A)$ as a $k$-vector space, which induces a map $\gamma: P \rightarrow \operatorname{gr}(A)$. It is immediate from the construction that $\operatorname{gr}(\tau)=\gamma$. In particular we see that $\operatorname{gr}(\tau)$ is independent of any choices. On the other hand, $\tau$ itself certainly depends on the choices of the formal coefficient field $K$ and formal coordinates $a_{i} \in \widehat{A}, i \in I$.

The following result addresses the dependence of $\tau$ on $K$ and $\left\{a_{i} \mid i \in I\right\}$ :

Proposition 3.9. Let $(A, \mathrm{~m}, k)$ be an equicharacteristic local ring, let $K, K^{\prime} \subset \widehat{A}$ be two formal coefficient fields and let $\left\{a_{i} \mid i \in I\right\} \subset \widehat{A}$ and $\left\{a_{i}^{\prime} \mid i \in I\right\} \subset \widehat{A}$ be two sets of formal coordinates. Consider the two maps $\tau: \widehat{P}:=k\left[\left[x_{i} \mid i \in I\right]\right] \rightarrow \widehat{A}$ and $\tau^{\prime}: \widehat{P}^{\prime}:=k\left[\left[x_{i}^{\prime} \mid i \in I\right]\right] \rightarrow \widehat{A}$ given by Proposition 3.7. Then there exists an isomorphism $\varphi: \widehat{P}^{\prime} \rightarrow \widehat{P}$ such that $\tau^{\prime}=\tau \circ \varphi$.

The proof of this proposition relies on the following basic property of formally smooth algebras. The statement is a natural generalisation of the definition of formal smoothness, which guarantees lifting not only via extensions with nilpotent kernel but also via extensions with topologically nilpotent kernel.

Proposition 3.10. Let $k_{0}$ be a topological ring and $k$ a formally smooth $k_{0}$-algebra. Let $C$ be a complete metrisable topological $k_{0}$-algebra and $\mathcal{I} \subset C$ a closed ideal such that $\left\{\mathcal{I}^{n}\right\}$ tends to zero. Then every continuous $k_{0}$-algebra homomorphism $u: k \rightarrow C / \mathcal{I}$ factors as $k \stackrel{v}{\rightarrow} C \rightarrow C / \mathcal{I}$, where $v$ is a continuous $k_{0}$-algebra homomorphism:

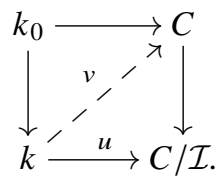

Proof. See [32, Chapter 0 0, Proposition 19.3.10].

Remark 3.11. Proposition 3.10 implies the existence of formal coefficient fields for any equicharacteristic local ring $(A, \mathrm{~m}, k)$. In this case, $C=\widehat{A}, \mathcal{I}=\widehat{\mathrm{m}}, k_{0}$ is the prime field contained in $\widehat{A}, k$ is the residue field and $u$ is the identity. Notice that $k_{0}$ is perfect, and therefore $k$ is separable over $k_{0}$ (hence formally smooth). Then $K=v(k)$ is a formal coefficient field.

Let $S$ be any discrete topological ring. For any two topological $S$-algebras $T$ and $T^{\prime}$, the tensor product $T \otimes_{S} T^{\prime}$ is endowed with the final topology with respect to its natural maps. The completed tensor product $T \hat{\otimes}_{S} T^{\prime}$ is defined to be the completion of $T \otimes_{S} T^{\prime}$. Note that the operation $\hat{\otimes}_{S}$ is the coproduct in the category of complete topological $S$-algebras.

Lemma 3.12. Let $(S, \mathfrak{n}, k)$ be a local $k$-algebra. Any continuous $S$-algebra map

$$
\varphi: S \hat{\otimes}_{k} k\left[\left[t_{i} \mid i \in I\right]\right] \rightarrow S \hat{\otimes}_{k} k\left[\left[z_{i} \mid i \in I\right]\right]
$$

which induces an isomorphism of continuous cotangent spaces is an isomorphism.

Proof. Note that a basis for the topology on $S \otimes_{k} k\left[\left[t_{i} \mid i \in I\right]\right]$ is given by the filtration

$$
\mathrm{m}_{n}:=\sum_{d+e=n} \mathfrak{n}^{d}+\left(\left(t_{i} \mid i \in I\right)^{e}\right) \widehat{.}
$$

Thus it follows that for the associated graded rings we have

$$
\operatorname{gr}\left(S \hat{\otimes}_{k} k\left[\left[t_{i} \mid i \in I\right]\right]\right) \simeq \operatorname{gr}\left(S \otimes_{k} k\left[t_{i} \mid i \in I\right]\right) \simeq \operatorname{gr}(S) \otimes_{k} k\left[t_{i} \mid i \in I\right] .
$$

The map $\varphi$ induces a $\operatorname{gr}(S)$-algebra map

$$
\operatorname{gr}(S)\left[t_{i} \mid i \in I\right] \rightarrow \operatorname{gr}(S)\left[z_{i} \mid i \in I\right],
$$


which by assumption is an isomorphism. Thus we can use [3, Lemma 10.23] to see that $\varphi$ is bijective. It is easy to check that $\varphi^{-1}$ is continuous, and we are done.

Proof of Proposition 3.9. Let $k_{0}$ be the prime field contained in $\widehat{A}$. Notice that $k$ is formally smooth over $k_{0}$. We apply Proposition 3.10 in the situation in which $C=\widehat{P}, \mathcal{I}=\operatorname{ker}(\tau)$ and $u: k \rightarrow \widehat{A}=C / \mathcal{I}$ is the map such that $u(k)=K^{\prime}$. Notice that $\mathcal{I}=\tau^{-1}(0)$ is closed because $\widehat{A}$ is separated, and that $\left\{\mathcal{I}^{n}\right\}$ tends to zero because $\mathcal{I}^{n} \subset \widehat{\mathfrak{\pi}^{n}}$. We get a map $v: k \rightarrow \widehat{P}$ verifying $\tau(v(k))=K^{\prime}$.

Since $\tau$ is surjective, there exist power series $f_{i} \in \widehat{P}$ such that $\tau\left(f_{i}\right)=a_{i}^{\prime}$. The map $\varphi$ is given by $\varphi\left(x_{i}^{\prime}\right)=f_{i}$ and $\left.\varphi\right|_{k}=v$. Lemma 3.12 shows that $\varphi$ is an isomorphism.

Remark 3.13. By the same argument as in the proof of Proposition 3.9, one can see that given any two formal embeddings $\tau: \widehat{P} \rightarrow \widehat{A}$ and $\tau^{\prime}: \widehat{P}^{\prime} \rightarrow \widehat{A}$ (not necessarily efficient), there is always a map $\varphi: \widehat{P}^{\prime} \rightarrow \widehat{P}$ such that $\tau^{\prime}=\tau \circ \varphi$, and if $\tau$ is efficient then $\varphi$ is surjective.

Proposition 3.14. For every equicharacteristic local ring $(A, \mathrm{~m}, k)$ we have

$$
\operatorname{edim}(A)=\min _{\tau} \operatorname{dim} \widehat{P}
$$

where the minimum is taken over all choices of formal embeddings $\tau: \widehat{P} \rightarrow \widehat{A}$ and is achieved whenever $\tau$ is an efficient formal embedding.

Proof. Let $\tau: \widehat{P} \rightarrow \widehat{A}$ be a formal embedding, and write $P=k\left[x_{i} \mid i \in I\right]$ and $\mathfrak{n}=\left(x_{i} \mid i \in I\right) \subset P$. Since $\tau$ is continuous, we have that $\tau\left(\widehat{\mathrm{n}}^{c}\right) \subset \widehat{\mathrm{m}}$ for some $c$. As $\widehat{\mathrm{m}}$ is maximal, this forces $\tau(\widehat{\mathrm{n}}) \subset \widehat{\mathrm{m}}$, and continuity gives $\tau\left(\widehat{\mathfrak{n}^{n}}\right) \subset \widehat{\mathrm{m}^{n}}$ for all $n$. Hence we get an induced map at the level of graded rings $\operatorname{gr}(\tau): P \rightarrow \operatorname{gr}(A)$. Since $\tau$ is surjective, $\operatorname{gr}(\tau)$ is also surjective and $\tau\left(\widehat{n^{n}}\right)=\widehat{m^{n}}$ for every $n$. In particular, $\tau$ induces a surjection at the level of Zariski cotangent spaces $\mathfrak{n} / \mathfrak{n}^{2} \rightarrow \mathfrak{m} / \mathfrak{m}^{2}$, and we see that $\operatorname{edim}(A) \leq \operatorname{dim} \widehat{P}$. If $\tau$ is an efficient formal embedding, then the map $\mathfrak{n} / \mathfrak{n}^{2} \rightarrow \mathfrak{m} / \mathfrak{m}^{2}$ is an isomorphism and we have $\operatorname{edim}(A)=\operatorname{dim} \widehat{P}$.

We finish this section by recalling the following result, which guarantees the existence of a Zariskilocal minimal embedding for singular points of a scheme of finite type over an infinite field. This is well known in the case of complex varieties (see, for example, [6, Theorem 3]) and we provide an extension of the proof to the more general case considered here.

Theorem 3.15. Let $X$ be a scheme of finite type over an infinite field $k$ and let $x \in X(k)$. If $\operatorname{edim}(X, x)=d$ and $X$ is not smooth at $x$, then there exist a closed subscheme $Y \subset \mathbb{A}_{k}^{d}$, a point $y \in Y(k)$ and an isomorphism

$$
\mathcal{O}_{Y, y} \simeq \mathcal{O}_{X, x}
$$

Proof. We may assume that $X$ is projective and embedded in $\mathbb{P}^{n}$ for some $n>d$. Denote by $\bar{k}$ the algebraic closure of $k$ and write $\bar{X}:=X \times_{k} \operatorname{Spec}(\bar{k})$ and $\bar{x}$ for the $\bar{k}$-point on $\bar{X}$ corresponding to $x$. As $\mathcal{O}_{\bar{X}, \bar{x}}$ is not a regular ring, we have

$$
\operatorname{dim}_{\bar{x}}(\bar{X})<\operatorname{edim}(\bar{X}, \bar{x})=\operatorname{edim}(X, x)=d .
$$

Suppose we can find a linear projection $\pi: \mathbb{P}^{n} \rightarrow \mathbb{P}^{d}$ defined over $k$ such that if $\bar{Y}$ denotes the schemetheoretic image of $\bar{X}$ under $\pi$ and $\bar{y}=\pi(\bar{x})$, then the induced map $\mathcal{O}_{\bar{Y}, \bar{y}} \rightarrow \mathcal{O}_{\bar{X}, \bar{x}}$ is an isomorphism. Since $\bar{Y}=Y \times_{k} \operatorname{Spec}(\bar{k})$, where $Y$ is the scheme-theoretic image of $X$ under the linear projection centred at $x$, we obtain a map $\mathcal{O}_{Y, y} \rightarrow \mathcal{O}_{X, x}$ whose base change to $\bar{k}$ gives the foregoing map. Thus, by faithfully flat descent, we get that $\mathcal{O}_{Y, y} \simeq \mathcal{O}_{X, x}$.

Now, in order to prove the claim, let $\bar{T} \subset \mathbb{P}_{\bar{k}}^{n}$ be the unique linear space passing through $\bar{x}$ whose tangent space at $\bar{x}$ agrees with that of $\bar{X}$. Furthermore, let $\bar{S}$ be the closure of the set of all lines connecting $\bar{z}$ with $\bar{x}$, where $\bar{z} \in \bar{X}, \bar{z} \neq \bar{x}$. Note that $\operatorname{dim}(\bar{S})=\operatorname{dim}(\bar{X})+1 \leq d$. Consider now the closure $\bar{Z}$ of the set 
$\bar{X} \cup \bar{T} \cup \bar{S}$, equipped with its reduced scheme structure. Since $\operatorname{dim}(\bar{S}) \leq d$ the set of all linear spaces $\bar{L}$ with $\bar{L} \cap \bar{Z}=\emptyset$ is open inside $\operatorname{Gr}(n-d-1, n) \times_{k} \operatorname{Spec}(\bar{k})$. The preimage of this set in $\operatorname{Gr}(n-d-1, n)$ is a nonempty open set, and since $k$ is infinite, it has a $k$-rational point, which we denote by $L$. Hence we have that the corresponding projection $\pi_{L}: \mathbb{P}^{n} \rightarrow \mathbb{P}^{d}$, defined over $k$, satisfies $\pi_{\bar{L}}^{-1}(\bar{y}) \cap \bar{X}=\{\bar{x}\}$ set-theoretically, where $\bar{y}$ corresponds to the $k$-point $y:=\pi_{L}(x)$. Writing $\bar{Y}:=\pi_{\bar{L}}(\bar{X})$, we get that the map of local rings $\mathcal{O}_{\bar{Y}, \bar{y}} \rightarrow \mathcal{O}_{\bar{X}, \bar{x}}$ is injective and finite. Since $\bar{L} \cap \bar{T}=\emptyset$, the tangent spaces of $\bar{x}$ and $\bar{y}$ are isomorphic and thus $\mathfrak{m}_{\bar{y}} \mathcal{O}_{\bar{X}, \bar{x}}=\mathfrak{m}_{\bar{x}}$. The claim now follows from the Nakayama lemma.

\section{Flatness of completion}

Let $A$ be a ring and $\mathrm{m}$ an ideal in $A$. Given an $A$-module $E$, we will consider the $\mathrm{m}$-adic topology on $E$ and we will denote by $\widehat{E}$ its $\mathrm{m}$-adic completion. We are interested in conditions guaranteeing that the natural map $A \rightarrow \widehat{A}$ is flat.

Definition 4.1. Let $E$ be an $A$-module and $F$ a submodule of $E$. We say that $F \subseteq E$ has the Artin-Rees property with respect to $\mathfrak{m}$ if there exists a $c \in \mathbb{N}$ such that for all $n>c$, we have

$$
\mathrm{m}^{n} E \cap F=\mathrm{m}^{n-c}\left(\mathrm{~m}^{c} E \cap F\right) .
$$

The smallest such $c$ is called the Artin-Rees index of $F \subseteq E$ with respect to $\mathrm{m}$. We say that $A$ has the Artin-Rees property with respect to $m$ if so does every finitely generated submodule of a finitely generated free $A$-module.

The Artin-Rees property for $F \subseteq E$ guarantees that the m-adic topology of $F$ coincides with the topology induced by the m-adic topology of $E$. In this context it is natural to consider the Rees algebra $A^{*}=\bigoplus_{n \geq 0} \mathrm{~m}^{n}$ and the graded $A^{*}$-modules

$$
E^{*}=\bigoplus_{n \geq 0} \mathfrak{m}^{n} E \quad \text { and } \quad F^{*}=\bigoplus_{n \geq 0} \mathfrak{m}^{n} E \cap F .
$$

Lemma 4.2. $F \subseteq E$ has the Artin-Rees property if and only if there exists a $c \in \mathbb{N}$ such that $F^{*}$ is generated as a graded $A^{*}$-module by elements of degree $\leq c$. Moreover, the Artin-Rees index of $F \subseteq E$ is the smallest such $c$.

Proof. This is immediate from the definitions. Compare with [7, Chapter III, Section 3.1, Theorem 1] or [36, Theorem 8.5] or [3, Lemma 10.8], but notice that no finite generation hypotheses are needed for the statement of the lemma.

Remark 4.3. By the classical Artin-Rees lemma [36, Theorem 8.5], any Noetherian ring $A$ has the Artin-Rees property with respect to any ideal $m \subset A$. By contrast, there exist non-Noetherian rings, even finite dimensional, which do not have the Artin-Rees property. A zero-dimensional example is given by

$$
A=k\left[x_{i} \mid i \in \mathbb{N}\right] /\left(x_{1}-x_{m}^{m} \mid m \geq 2\right)+\left(x_{n}^{n+1} \mid n \geq 1\right),
$$

with $\mathrm{m}=\left(x_{i} \mid i \in \mathbb{N}\right)$ and $F=\left(x_{1}\right) \subset E=A$. Clearly $x_{1} \in \mathfrak{m}^{n}$ for all $n$, but there is no $f \in \mathfrak{m}$ such that $x_{1}=x_{1} f$.

In complete analogy with the Noetherian case, we prove that the Artin-Rees property implies flatness of the completion. We recall that a ring is coherent if every finitely generated ideal is finitely presented.

Proposition 4.4. Let $A$ be a coherent ring with the Artin-Rees property with respect to $\mathrm{m} \subset A$, and let $\widehat{A}$ be its $\mathbf{m}$-adic completion. Then $A \rightarrow \widehat{A}$ is flat. Moreover, if $\mathfrak{a} \subset A$ is a finitely generated ideal, then $\mathfrak{a} \widehat{A}$ is closed in $\widehat{A}$ (that is, $\mathfrak{a} \widehat{A}=\widehat{\mathfrak{a}}$ ). 
Proof. Let $\mathfrak{a}$ be a finitely generated ideal of $A$. Since $A$ is coherent, there exists an exact sequence

$$
A^{p} \longrightarrow A^{q} \stackrel{\varphi}{\longrightarrow} \mathfrak{a} \longrightarrow 0 .
$$

Moreover, since the Artin-Rees property holds for $\operatorname{ker} \varphi \subset A^{q}$, the m-adic topology on $\operatorname{ker} \varphi$ agrees with the one induced by the inclusion $\operatorname{ker} \varphi \subset A^{q}$. From [7, Chapter III, Section 2.12, Lemma 2] or [3, Lemma 10.3], the sequence remains exact after taking $m$-adic completions, and we have a commutative diagram

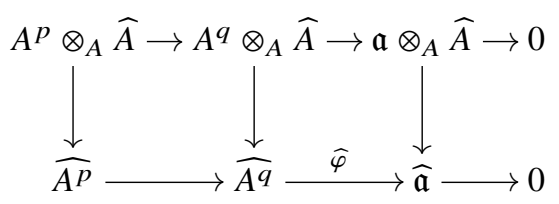

with exact rows. Since taking completion commutes with finite direct sums, the map $\mathfrak{a} \otimes_{A} \widehat{A} \rightarrow \widehat{\mathfrak{a}}$ is an isomorphism. As the natural map $\widehat{\mathfrak{a}} \rightarrow \widehat{A}$ is an injection, the flatness of $A \rightarrow \widehat{A}$ follows from [36, Theorem 7.7]. The fact that $\mathfrak{a} \otimes_{A} \widehat{A} \rightarrow \widehat{\mathfrak{a}}$ is an isomorphism also shows that $\mathfrak{a} \widehat{A}=\widehat{\mathfrak{a}}$.

The following theorem gives a first example of a non-Noetherian ring with the Artin-Rees property. We were not able to find a reference for this statement in the literature.

Theorem 4.5. Let $S$ be a Noetherian ring and $\mathrm{n}$ any ideal of $S$. For any set $I$, consider $P=S\left[x_{i} \mid i \in I\right]$ and $\mathrm{m}=\left(x_{i} \mid i \in I\right)+\mathfrak{n}$. Then $P$ has the Artin-Rees property with respect to $\mathrm{m}$.

Proof. Let $E$ be a finitely generated free $P$-module and $F \subseteq E$ a finitely generated submodule. Assume that $E$ is freely generated by $e_{1}, \ldots, e_{s}$.

Given any subset $J \subseteq I$, we write $P_{J}:=S\left[x_{i} \mid i \in J\right]$, and for any ideal $\mathfrak{a} \subseteq P$ we denote $\mathfrak{a}_{J}:=\mathfrak{a} \cap P_{J}$. We define $E_{J}:=P_{J} \cdot e_{1} \oplus \cdots \oplus P_{J} \cdot e_{S}$, and for any $P$-submodule $G \subseteq E$ we write $G_{J}:=E_{J} \cap G$. Note that $P, \mathfrak{m}, \mathfrak{a}, E, G$ are the colimits of $P_{J}, \mathfrak{m}_{J}, \mathfrak{a}_{J}, E_{J}, G_{J}$ for $J \subseteq I$ finite. We have

$$
G_{J} \cap G_{J}^{\prime}=\left(G \cap G^{\prime}\right)_{J}, \quad \mathfrak{a}_{J} G_{J} \subseteq(\mathfrak{a} G)_{J}, \quad \mathfrak{a}_{J} E_{J}=(\mathfrak{a} E)_{J}, \quad\left(\mathfrak{m}_{J}\right)^{n}=\left(\mathfrak{m}^{n}\right)_{J} .
$$

In particular, for all $n, d \in \mathbb{N}$ with $n>d$, we have

$$
\mathrm{m}_{J}^{n} E_{J} \cap F_{J}=\left(\mathrm{m}^{n} E \cap F\right)_{J} \quad \text { and } \quad \mathrm{m}_{J}^{n-d}\left(\mathrm{~m}_{J}^{d} E_{J} \cap F_{J}\right) \subseteq\left(\mathrm{m}^{n-d}\left(\mathrm{~m}^{d} E \cap F\right)\right)_{J} .
$$

Assume that $F$ is generated by $f_{1}, \ldots, f_{r}$. Then there exists a finite set $L \subset I$ such that $f_{1}, \ldots, f_{r} \in F_{L}$, and for any $J$ with $L \subseteq J \subseteq I$ we have $F_{J}=P_{J} \cdot f_{1}+\cdots+P_{J} \cdot f_{r}=P_{J} \cdot F_{L}$.

Since $P_{L}$ is Noetherian, it has the Artin-Rees property with respect to $\mathfrak{m}_{L}$, and hence there exists a $c \in \mathbb{N}$ such that

$$
\mathrm{m}_{L}^{n} E_{L} \cap F_{L}=\mathrm{m}_{L}^{n-c}\left(\mathrm{~m}_{L}^{c} E_{L} \cap F_{L}\right)
$$

for all $n>c$. The smallest such $c$ is the Artin-Rees index of $F_{L} \subseteq E_{L}$. Since for any finite set $J$ with $L \subseteq J \subset I$ we have $F_{J}=P_{J} \cdot F_{L}$, we can apply Lemma 4.6, and we see that the Artin-Rees index of $F_{J} \subseteq E_{J}$ is again $c$. This implies that

$$
\left(\mathfrak{m}^{n} E \cap F\right)_{J} \subseteq\left(\mathfrak{m}^{n-c}\left(\mathfrak{m}^{c} E \cap F\right)\right)_{J} .
$$

Taking the colimit for all finite $J \subset I$, we get

$$
\mathrm{m}^{n} E \cap F \subseteq \mathrm{m}^{n-c}\left(\mathrm{~m}^{c} E \cap F\right) .
$$

The reversed inclusion is immediate, and the theorem follows. 
Lemma 4.6. Let $A_{0}$ be a Noetherian ring, $\mathrm{m}_{0} \subset A_{0}$ an ideal, $E_{0}$ a finitely generated $A_{0}$-module and $F_{0} \subseteq E_{0}$ a submodule. Let $z$ be a new variable and consider the ring $A=A_{0}[z]$, the ideal $\mathrm{m}=\mathrm{m}_{0} A+(z)$, the extension $E=A \otimes_{A_{0}} E_{0}=E_{0}[z]$ and $F=A \otimes_{A_{0}} F_{0}=F_{0}[z]$. Then the Artin-Rees index of $F \subseteq E$ with respect to $\mathrm{m}$ equals the Artin-Rees index of $F_{0} \subseteq E_{0}$ with respect to $\mathbf{m}_{0}$.

Proof. Let $c_{0}$ and $c$ be the Artin-Rees indexes of $F_{0} \subseteq E_{0}$ and $F \subseteq E$. As in Lemma 4.2, consider the Rees algebras

$$
A_{0}^{*}=\bigoplus_{n \geq 0} \mathrm{~m}_{0}^{n} \quad \text { and } \quad A^{*}=\bigoplus_{n \geq 0} \mathrm{~m}^{n}
$$

and the graded modules

$$
F_{0}^{*}=\bigoplus_{n \geq 0} \mathrm{~m}_{0}^{n} E_{0} \cap F_{0} \quad \text { and } \quad F^{*}=\bigoplus_{n \geq 0} \mathrm{~m}^{n} E \cap F .
$$

Then $F_{0}$ is generated in degree $\leq c_{0}$ as a graded $A_{0}$-algebra (and not in any lower degree), and similarly for $F$.

Any element $f \in \mathfrak{m}^{n} E \cap F$ can be written as $f=\sum_{i=0}^{n} f_{i} z^{n-i}$, where $f_{i} \in \mathfrak{m}_{0}^{i} E_{0} \cap F_{0}$. In particular, $F^{*}$ is generated by $F_{0}^{*}$ as an $A^{*}$-algebra, and therefore $c \leq c_{0}$. Conversely, if $F^{*}$ is generated by homogeneous elements $f^{(1)}, \ldots, f^{(r)}$ with $f^{(j)}=\sum_{i} f_{i}^{(j)} z^{n_{j}-i}$, then $F_{0}^{*}$ is generated by $f_{n_{1}}^{(1)}, \ldots, f_{n_{r}}^{(r)}$. We see that $c_{0} \leq c$, and the result follows.

Remark 4.7. If $A$ and $m$ are as in Remark 4.3, then we have $A=\lim _{\longrightarrow} A_{m}$, where

$$
A_{m}=k\left[x_{1}, \ldots, x_{m}\right] /\left(x_{1}-x_{i}^{i}, x_{i}^{i+1}\right), \quad 1<i \leq m .
$$

It is easy to check that the Artin-Rees index of $\left(x_{1}\right) \subset A_{m}$ is $m$ and $A$ does not have the Artin-Rees property.

Recall that for any discrete topological ring $S$ and any two topological $S$-algebras $T$ and $T^{\prime}$, the completed tensor product $T \hat{\otimes}_{S} T^{\prime}$ is defined to be the completion of $T \otimes_{S} T^{\prime}$ with respect to its natural topology.

Corollary 4.8. Let $S \rightarrow T$ be a map of Noetherian rings. As before, suppose that $S$ has the discrete topology, and let $T$ be equipped with the $\mathfrak{n}$-adic topology where $\mathfrak{n} \subset T$ is an ideal. Then the natural map

$$
T\left[x_{i} \mid i \in I\right]=T \otimes_{S} S\left[x_{i} \mid i \in I\right] \rightarrow T \hat{\otimes}_{S} S\left[x_{i} \mid i \in I\right]
$$

is flat. In particular:

1. for any index set $I$, the completion map $S\left[x_{i} \mid i \in I\right] \rightarrow S\left[\left[x_{i} \mid i \in I\right]\right]$ is flat and

2. for every finite subset $J \subset I$, the inclusion $S\left[\left[x_{j} \mid j \in J\right]\right] \rightarrow S\left[\left[x_{i} \mid i \in I\right]\right]$ is flat.

Proof. Observe that a basis for the topology on $T\left[x_{i} \mid i \in I\right]$ is given by

$$
\mathfrak{n}^{m}\left[x_{i} \mid i \in I\right]+\left(x_{i} \mid i \in I\right)^{n}, \quad m, n \in \mathbb{N},
$$

which is easily seen to be equivalent to the $\mathrm{m}$-adic one, where $\mathrm{m}:=\left(x_{i} \mid i \in I\right)+\mathfrak{n}$. As $T\left[x_{i} \mid i \in I\right]$ is coherent (see, for example, [30, Theorem 6.2.2]), the first assertion follows from Theorem 4.5 and Proposition 4.4. Regarding the last two assertions, (1) follows by observing that $S \hat{\otimes}_{S} S\left[x_{i} \mid i \in I\right]=$ $S\left[\left[x_{i} \mid i \in I\right]\right]$, and (2) by taking $T=S\left[\left[x_{j} \mid j \in J\right]\right]$ with the $\left(x_{j} \mid j \in J\right)$-adic topology and observing that the given inclusion factors as

$$
S\left[\left[x_{j} \mid j \in J\right]\right] \rightarrow S\left[\left[x_{j} \mid j \in J\right]\right]\left[x_{i} \mid i \in I \backslash J\right] \rightarrow S\left[\left[x_{i} \mid i \in I\right]\right],
$$

and so is flat. 
Remark 4.9. For quotients $A$ of $k\left[x_{i} \mid i \in I\right]$, the completion map $A \rightarrow \widehat{A}$ need not be flat, even if the topology of $A$ is separated. Consider the ideal

$$
\mathfrak{a}=\left(y x_{1}, y x_{n}^{n}-z x_{n-1}^{n-1} \mid n>1\right)
$$

in $P=k\left[x_{n}, y, z \mid n \in \mathbb{N}_{>0}\right]$ and the quotient $A=P / \mathfrak{a}$. Let $\mathfrak{m}=\left(x_{n}, y, z \mid n \in \mathbb{N}_{>0}\right) \subset A$. As $\mathfrak{m}$ is weighted homogeneous with respect to the positive weights $w\left(x_{n}\right)=w(y)=1, w(z)=2$, it follows that the m-adic topology on $A$ is separated. Consider the element $y-z$, which is annihilated by the series $f=\sum_{n \geq 1} x_{n}^{n}$. If $\widehat{A}$ were flat over $A$, there would exist polynomials $a_{1}, \ldots, a_{r} \in A$ annihilating $y-z$ such that $f$ could be written as $f=\sum_{j=1}^{r} a_{j} b_{j}$, where $b_{j} \in \widehat{A}$.

Considering this equation modulo $(y, z)$, we have written $f$ as a linear combination of polynomials in $k\left[x_{n} \mid n \in \mathbb{N}_{>0}\right]$, which is clearly impossible.

We close this section with the following analogue to Proposition 2.7 for polynomial rings:

Proposition 4.10. Let $P=S\left[x_{i} \mid i \in I\right]$ and $\mathrm{m}=\left(x_{i} \mid i \in I\right)$, where $S$ is a ring and $I$ a set. Let $\mathfrak{a} \subset P_{\mathfrak{m}}$ be an ideal such that in $(\mathfrak{a}) \subset P$ is finitely generated. Then $\mathfrak{a}$ is finitely generated.

Proof. Let $f_{1}, \ldots, f_{r} \in \mathfrak{a}$ be such that $\operatorname{in}\left(f_{1}\right), \ldots, \operatorname{in}\left(f_{r}\right)$ generate in $(\mathfrak{a})$. Since in $(\mathfrak{a})=\operatorname{in}(\widehat{\mathfrak{a}})$, we can apply Proposition 2.7 to see that $\widehat{\mathfrak{a}}=\left(f_{1}, \ldots, f_{r}\right) \widehat{P}$. By Corollary 4.8, the map $P_{\mathfrak{m}} \rightarrow \widehat{P}$ is faithfully flat and thus $\mathfrak{a} \subset \widehat{\mathfrak{a}} \cap P_{\mathfrak{m}}=\left(f_{1}, \ldots, f_{r}\right) P_{\mathfrak{m}}$. The other inclusion is trivial, so $\mathfrak{a}=\left(f_{1}, \ldots, f_{r}\right)$.

\section{Ideals of finite definition}

In this section, we fix a field $k$ and a set $I$ and consider the polynomial $\operatorname{ring} P=k\left[x_{i} \mid i \in I\right]$ and the power series ring $\widehat{P}=k\left[\left[x_{i} \mid i \in I\right]\right]$. An important class of ideals in $\widehat{P}$ are those generated by finitely many power series involving only finitely many variables. We study their properties in this section.

For any subset $J \subset I$, we write $P_{J}=k\left[x_{i} \mid i \in J\right]$ and $\widehat{P}_{J}=k\left[\left[x_{i} \mid i \in J\right]\right]$, and for any ideal $\mathfrak{a} \subset \widehat{P}$ we denote $\mathfrak{a}_{J}:=\mathfrak{a} \cap \widehat{P}_{J}$.

Definition 5.1. Let $\mathfrak{a} \subset \widehat{P}$ be an ideal.

1. We say that $\mathfrak{a}$ is of finite definition with respect to the indeterminates $x_{i}$ if there exists a finite subset $J \subset I$ such that $\mathfrak{a}=\mathfrak{a}_{J} \widehat{P}$.

2. Similarly, $\mathfrak{a}$ is of finite polynomial definition with respect to the indeterminates $x_{i}$ if it is generated by finitely many polynomials - that is, elements in $P$.

3. We say that $\mathfrak{a}$ is of finite (polynomial) definition if there exists a $k$-isomorphism $\widehat{P} \simeq k\left[\left[x_{i}^{\prime} \mid i \in I\right]\right]$ such that $\mathfrak{a}$ is of finite (polynomial) definition with respect to the formal coordinates $x_{i}^{\prime}$.

Definition 5.2. Let $(A, \mathrm{~m}, k)$ be an equicharacteristic local ring.

1. A weak DGK decomposition for $A$ is an isomorphism $\widehat{A} \simeq k\left[\left[x_{i} \mid i \in I\right]\right] / \mathfrak{a}$, where $\mathfrak{a}$ is an ideal of finite definition.

2. A $D G K$ decomposition for $A$ is an isomorphism $\widehat{A} \simeq k\left[\left[x_{i} \mid i \in I\right]\right] / \mathfrak{a}$ with $\mathfrak{a}$ of finite polynomial definition.

3. We say that a (weak) DGK decomposition $\widehat{A} \simeq k\left[\left[x_{i} \mid i \in I\right]\right] / \mathfrak{a}$ is efficient if the quotient map $k\left[\left[x_{i} \mid i \in I\right]\right] \rightarrow \widehat{A}$ is an efficient formal embedding.

Remark 5.3. If $A$ has a DGK decomposition, then we have an isomorphism $\widehat{A} \simeq \widehat{B} \hat{\otimes}_{k} \widehat{P}$, where $\widehat{P}$ is a power series ring and $(B, \mathfrak{n}, k)$ is a local $k$-algebra which is essentially of finite type. Geometrically, this means that $\operatorname{Spf}(\widehat{A}) \simeq \widehat{Z}_{z} \widehat{\times} \Delta^{I}$, where $\Delta^{I}=\operatorname{Spf}\left(k\left[\left[x_{i} \mid i \in I\right]\right]\right)$ and $\widehat{Z}_{z}$ is the formal neighbourhood of a scheme $Z$ of finite type over $k$ at a point $z \in Z(k)$. If $A$ has a weak DGK decomposition, then $\widehat{A} \simeq \mathcal{B} \hat{\otimes}_{k} \widehat{P}$, where $\mathcal{B}$ is a Noetherian complete local ring with residue field $k$. 
Example 5.4. The existence of a weak DGK decomposition for a ring $A$ does not imply the existence of a DGK decomposition for $A$. This can be seen by considering the following example given by Whitney. Let $f(t)$ be a transcendental power series with complex coefficients and with $f(0)=0$, and consider the equation

$$
g=x y(y-x)(y-(3+t) x)(y-(4+f(t)) x) .
$$

It is proven in [46, Example 14.1] that $\mathcal{B}=\mathbb{C}[[x, y, t]] /(g)$ is not isomorphic to the completion of a local ring of a $\mathbb{C}$-scheme of finite type. In particular, any local $\operatorname{ring} A$ for which $\widehat{A} \simeq \mathcal{B}$ (for example, $\mathcal{B}$ itself) admits a weak DGK decomposition but not a DGK decomposition.

We now give another example of a local $\operatorname{ring} A$ such that $\widehat{A} \simeq \mathcal{B}$. This example has the advantage of being explicitly presented as the localisation of a quotient of a polynomial ring in countably many variables. Write $f(t)=\sum_{i \geq 1} a_{i} t^{i} \in \mathbb{C}[[t]]$. Consider the polynomial ring $P=\mathbb{C}\left[x, y, t, z_{n} \mid n \geq 0\right]$ and the ideal

$$
\mathfrak{a}=\left(h, z_{n-1}-z_{n} t-a_{n} t \mid n \geq 1\right),
$$

where

$$
h=x y(y-x)(y-(3+t) x)\left(y-\left(4+z_{0}\right) x\right) .
$$

Let $A$ be the localisation of $P / \mathfrak{a}$ at the ideal $\left(x, y, t, z_{n} \mid n \geq 0\right)$. Then in $\widehat{A}$ we have, for each $m \geq 1$,

$$
z_{0}-f(t)=z_{m} t^{m}-\sum_{i \geq m+1} a_{i} t^{i} \in \widehat{\mathrm{m}}^{m}
$$

and for each $m \geq n+1$,

$$
z_{n}-\sum_{i \geq n+1} a_{i} t^{i-n}=z_{m} t^{m-n}-\sum_{i \geq m+1} a_{i} t^{i-n} \in \widehat{\mathrm{m}}^{m-n} .
$$

Thus it follows that $\widehat{A} \simeq \mathbb{C}\left[\left[x, y, t, z_{0}\right]\right] /\left(h, z_{0}-f(t)\right) \simeq \mathbb{C}[[x, y, t]] /(g)=\mathcal{B}$.

Remark 5.5. An analogous definition of finite definition can be given for ideals in a polynomial ring $P=k\left[x_{i} \mid i \in I\right]$. It is easy to see that the definition does not depend on the choice of indeterminates, and that an ideal of $P$ is of finite definition if and only if it is finitely generated. By contrast, in a power series ring not every ideal of finite definition is so with respect to the given indeterminates $x_{i}$, and not every finitely generated ideal is of finite definition. For instance, consider $\widehat{P}=k\left[\left[x_{n} \mid n \in \mathbb{N}\right]\right]$. The principal ideal generated by $f=\sum_{n \geq 1} x_{n}^{n}$ is of finite definition by Lemma 3.12 but not in the indeterminates $x_{i}$. As for the second claim, an example is given by the principal ideal generated by $g=\sum_{n \geq 1} x_{n}^{n+1}$, which, as we shall discuss next, is not of finite definition if $k$ is of characteristic 0 . Indeed, assume by contradiction that there exists an isomorphism $\widehat{P} \simeq k\left[\left[y_{n} \mid n \in \mathbb{N}\right]\right]$ such that $g \widehat{P}$ is of finite definition with respect to the indeterminates $y_{n}$. Pick a variable $y_{r}$ not appearing in the generators for $g \widehat{P}$, and consider the regular continuous derivation $d=\partial / \partial y_{r}$ on $\widehat{P}$. Notice that $d(g)=0$. By regularity, we have $d\left(x_{m}\right) \in \widehat{P}^{\times}$for some $m \geq 1$. Writing $d(g)=\sum_{n \geq 1}(n+1) x_{n}^{n} d\left(x_{n}\right)$, we see that $\operatorname{ord}_{x_{m}}(d(g))<\infty$, contradicting $d(g)=0$.

Ideals of finite definition form a class of ideals of $\widehat{P}$ for which Question 2.8 has a positive answer. We give only a sketch of the proof of the next lemma here, and refer the reader to [18, Section 1.5] for details.

Lemma 5.6. Let $\mathfrak{a} \subset \widehat{P}$ be an ideal such that there exists $J \subset$ I finite with $\mathfrak{a}=\mathfrak{a}_{J} \widehat{P}$. Then in $(\mathfrak{a})=\operatorname{in}\left(\mathfrak{a}_{J}\right) P$.

Proof. Choose a local monomial order $\prec$ compatible with the standard filtration on $\widehat{P}$; for example, take the order defined by $x^{a}<x^{b}$ if $x^{b}<$ grlex $x^{a}$, where $<_{\text {grlex }}$ denotes the usual graded lexicographic order. Then $\prec$ restricts to a local monomial order $\prec_{J}$ on $\widehat{P}_{J}$. Choose a standard basis $S=\left\{f_{1}, \ldots, f_{r}\right\}$ 
of $\mathfrak{a}_{J}$ with respect to $\prec_{J}$. By [4, Theorem 4.1], this is equivalent to $S$ being closed under $s$-series. Note that $[4$, Theorem 4.1] extends directly to the case of infinitely many indeterminates, and thus it follows immediately that $S$ is a standard basis of $\mathfrak{a}$ with respect to $\prec$. Clearly we have in $(\mathfrak{a})=\left(\operatorname{in}\left(f_{1}\right), \ldots, \operatorname{in}\left(f_{r}\right)\right)$, which proves the claim.

We are interested now in understanding heights of ideals of finite definition. Let us start by looking at their minimal primes.

Proposition 5.7. If $\mathfrak{a} \subset \widehat{P}$ is an ideal of finite definition, then it has a finite number of minimal primes, and each of them is of finite definition. More precisely, let $J \subset I$ be a finite subset and assume that $\mathfrak{a}=\mathfrak{a}_{J} \widehat{P}$. If $\mathfrak{p} \subset \widehat{P}$ is a minimal prime of $\mathfrak{a}$, then $\mathfrak{p}=\mathfrak{p}_{J} \widehat{P}$. Moreover, the assignment $\mathfrak{p} \mapsto \mathfrak{p}_{J}$ gives $a$ bijection between the minimal primes of $\mathfrak{a}$ and the minimal primes of $\mathfrak{a}_{J}$.

Proof. Notice that if $\mathfrak{p} \subset \widehat{P}$ is a prime ideal, then $\mathfrak{p}_{J} \subset \widehat{P}_{J}$ remains prime. Moreover, by Corollary 4.8 we have that $\widehat{P}_{J} \rightarrow \widehat{P}$ is faithfully flat and thus $\mathfrak{q}=(\mathfrak{q} \widehat{P}) \cap \widehat{P}_{J}$ for any ideal $\mathfrak{q} \subset \widehat{P}_{J}$. It is therefore sufficient to show that for every prime ideal $\mathfrak{q} \subset \widehat{P}_{J}$, the extension $\mathfrak{q} \widehat{P}$ is prime. By Remark 2.4 we have an injection $\widehat{P} \rightarrow\left(\widehat{P}_{J}\right)^{\mathbb{N}^{(I \backslash J)}}$. Since $J$ is finite, $\widehat{P}_{J}$ is Noetherian and $\mathfrak{q}$ is finitely generated. This implies that $\mathfrak{q}\left(\widehat{P}_{J}\right)^{\mathbb{N}^{(I \backslash J)}}=\mathfrak{q}^{\mathbb{N}^{(I \backslash J)}}$ - that is, the elements of the extension $\mathfrak{q}\left(\widehat{P}_{J}\right)^{\mathbb{N}^{(I \backslash J)}}$ are precisely the Bourbaki power series that, when expanded in the variables indexed by $I \backslash J$, have coefficients in $\mathfrak{q}$. Therefore we have an injection

$$
\widehat{P} / \mathfrak{q} \widehat{P} \hookrightarrow\left(\widehat{P}_{J} / \mathfrak{q}\right)^{\mathbb{N}^{(I \backslash J)}}
$$

and the ring in the right-hand side is clearly a domain. Thus $\mathfrak{q} \widehat{P}$ is prime.

Remark 5.8. In the setup of the proof of Proposition 5.7, if $J$ is infinite then it is no longer true that $\mathfrak{q}\left(\widehat{P}_{J}\right)^{\mathbb{N}^{(I \backslash J)}}=\mathfrak{q}^{\mathbb{N}^{(I \backslash J)}}$ for an arbitrary prime $\mathfrak{q} \subset \widehat{P}_{J}$. For example, let $J=\mathbb{N}$, pick $i_{0} \in I \backslash J$, let $\mathfrak{q}=\widehat{\mathrm{m}}_{J}$ be the maximal ideal in $\widehat{P}_{J}$ and consider the series $f=\sum_{n \in \mathbb{N}} x_{n} x_{i_{0}}^{n}$. Then $f$ belongs to $\mathfrak{q}^{\mathbb{N}^{(I \backslash J)}}$ but not to $\mathfrak{q}\left(\widehat{P}_{J}\right)^{\mathbb{N}^{(I \backslash J)}}$. We do not know if the extension $\mathfrak{q} \widehat{P}$ remains prime when $J$ is infinite.

Remark 5.9. Proposition 5.7 shows that the ideal $\left(x_{i} \mid i \in J\right) \widehat{P}$ is prime whenever $J$ is finite. Since colimits of prime ideals remain prime, one sees that $\left(x_{i} \mid i \in J\right) \widehat{P}$ is prime for an arbitrary subset $J$. In particular, $\mathrm{m}_{0}=\left(x_{i} \mid i \in I\right) \widehat{P}$ is prime. Notice that $\widehat{P} / \mathrm{m}_{0}$ has infinite dimension when $I$ is infinite.

The proof of the following theorem uses the results of the previous section and Proposition 5.13:

Theorem 5.10. If $J \subset I$ is a finite subset and $\mathfrak{a}=\mathfrak{a}_{J} \widehat{P}$, then $\operatorname{ht}(\mathfrak{a})=\operatorname{ht}\left(\mathfrak{a}_{J}\right)$.

Proof. From Proposition 5.7 we can assume that $\mathfrak{a}=\mathfrak{p}=\mathfrak{p}_{J} \widehat{P}$ is a prime ideal. Notice that $\mathfrak{p} \subset \mathfrak{b}:=\left(x_{j} \mid\right.$ $j \in J)$. From Proposition 5.13, the localisation $\widehat{P}_{\mathfrak{b}}$ is Noetherian, and therefore $\widehat{P}_{\mathfrak{p}}$, which is a further localisation of $\widehat{P}_{\mathrm{b}}$, is also Noetherian. By Corollary 4.8, the extension $\widehat{P}_{J} \subset \widehat{P}$ is flat, and therefore the extension $\left(\widehat{P}_{J}\right)_{\mathfrak{p}_{J}} \subset \widehat{P}_{\mathfrak{p}}$ is also flat. Since $\widehat{P}_{\mathfrak{p}}$ is Noetherian, it follows from [36, Theorem 15.1] that $\operatorname{ht}(\mathfrak{p})=\operatorname{ht}\left(\mathfrak{p}_{J}\right)$.

Corollary 5.11. Let $\mathfrak{a} \subset \widehat{P}$ be any ideal of finite definition. For every minimal prime $\mathfrak{p}$ of $\mathfrak{a}$, we have $\operatorname{ht}(\mathfrak{p})<\infty$.

Remark 5.12. In the case of polynomial rings, the analogues of Theorem 5.10 and Corollary 5.11 are well known and easy to prove, and in fact there is a strong converse to the analogue of Corollary 5.11, since every prime ideal of finite height in a polynomial ring $P=k\left[x_{i} \mid i \in I\right]$ is finitely generated. To see this, suppose $\mathfrak{p} \subset k\left[x_{i} \mid i \in I\right]$ is a prime ideal that is not finitely generated. Recall that $\mathfrak{p}$ is the 
colimit of the ideals $\mathfrak{p} \cap P_{J}$ as $J$ ranges among the finite subsets of $I$. This implies that we can fix an embedding $\mathbb{N} \subset I$ and find an increasing sequence $\left\{r_{n} \mid n \in \mathbb{N}\right\} \subset \mathbb{N}$ such that if $\mathfrak{p}_{n} \subset k\left[x_{i} \mid i \in I\right]$ is the ideal generated by $\mathfrak{p} \cap k\left[x_{1}, \ldots, x_{r_{n}}\right]$, then $\mathfrak{p}_{n} \subsetneq \mathfrak{p}_{n+1}$ for all $n$. Since $\mathfrak{p}_{n}$ are all prime and are contained in $\mathfrak{p}$, it follows that ht $(\mathfrak{p})=\infty$.

Moreover, for arbitrary ideals $\mathfrak{a}$ of $P$ it is proven in [29, Theorem 3.3] that $\mathfrak{a}$ is finitely generated if and only if it has finitely many associated primes, each of which is of finite height.

Proposition 5.13. For every finite $J \subset I$, the localisation $\widehat{P}_{\left(x_{j} \mid j \in J\right)}$ is Noetherian.

Proof. As discussed in Remark 2.5, since $J$ is finite we have an isomorphism

$$
\widehat{P} \simeq k\left[\left[x_{i} \mid i \in I \backslash J\right]\right]\left[\left[x_{j} \mid j \in J\right]\right]
$$

The proposition now follows from the next lemma:

Lemma 5.14. For any $n \in \mathbb{N}$, let $\widehat{P}_{n}:=\widehat{P}\left[\left[y_{1}, \ldots, y_{n}\right]\right]$ and consider the ideal $\mathfrak{b}_{n}:=\left(y_{1}, \ldots, y_{n}\right)$ in $\widehat{P}_{n}$. Then the localisation $\left(\widehat{P}_{n}\right)_{\mathfrak{b}_{n}}$ is a Noetherian ring.

The proof of this lemma uses the following straightforward generalisation of the Weierstrass division theorem, whose proof is a simple adaptation of the proof of [7, VII, Section 3.8] where the adic topology on $\widehat{P}_{n}$ is replaced with the inverse limit topology. We say that $f \in \widehat{P}_{n}$ is $y_{n}$-regular of order $d$ if its image under the canonical map $\widehat{P}_{n} \rightarrow k\left[\left[y_{n}\right]\right]$ is nonzero of order $d$.

Theorem 5.15. Let $f \in \widehat{P}_{n+1}$ be $y_{n+1}$-regular of order $d$. For every $g \in \widehat{P}_{n+1}$ there exist unique $q \in \widehat{P}_{n+1}$ and $r \in \widehat{P}_{n}\left[y_{n+1}\right]$ such that $g=q f+r$ and $r$ has degree $<r$ as a polynomial in $y_{n+1}$.

The next lemma ensures that we can apply Theorem 5.15 to prove Lemma 5.14.

Lemma 5.16. Let $f \in \widehat{P}_{n+1}=\widehat{P}\left[\left[y_{1}, \ldots, y_{n+1}\right]\right]$ be a nonzero element. Then there exists a continuous k-automorphism $\varphi: \widehat{P}_{n+1} \rightarrow \widehat{P}_{n+1}$ such that $\varphi\left(\mathfrak{b}_{n+1}\right)=\mathfrak{b}_{n+1}$ and $\varphi(f)$ is $y_{n+1}$-regular.

Proof. If $f$ is already $y_{n+1}$-regular, then we are done. If not, then pick any monomial of the form $x_{i_{1}}^{d_{1}} \cdots x_{i_{r}}^{d_{r}} y_{1}^{e_{1}} \cdots y_{n+1}^{e_{n+1}}$ appearing in the expansion of $f$. Then decompose $f$ as

$$
f=f^{\prime}+f^{\prime \prime}, \quad f^{\prime} \in k\left[\left[x_{i_{1}}, \ldots, x_{i_{r}}, y_{1}, \ldots, y_{n+1}\right]\right]
$$

such that $f^{\prime}$ cannot be decomposed further. By [1, Lemma 6.11] there exist new coordinates $x_{i_{j}}^{\prime}=$ $x_{i_{j}}+y_{n+1}^{a_{j}}, y_{l}^{\prime}=y_{l}+y_{n+1}^{b_{l}}$ and $y_{n+1}^{\prime}:=y_{n+1}$ such that $f^{\prime}\left(x_{i_{j}}^{\prime}, y_{l}^{\prime}\right)$ is $y_{n+1}^{\prime}$-regular. We may extend this change of coordinates trivially to a continuous automorphism $\varphi: \widehat{P}_{n+1} \rightarrow \widehat{P}_{n+1}$ by setting $x_{i}^{\prime}=x_{i}$ for all indices $i$ that are different from $i_{j}$ for all $j$. Then clearly $\varphi(f)$ is $y_{n+1}$-regular and $\varphi$ fixes $\mathfrak{b}_{n+1}=\left(y_{1}, \ldots, y_{n+1}\right)$.

Proof of Lemma 5.14. We prove the lemma by induction on $n$. Let $Q_{n}:=\left(\widehat{P}_{n}\right)_{\mathfrak{b}_{n}}$. Clearly $Q_{0} \simeq \operatorname{Quot}(\widehat{P})$, so let us assume that $Q_{n}$ is Noetherian. We have injections $Q_{n} \rightarrow Q_{n+1}$. Let $\mathfrak{a}$ be an ideal of $Q_{n+1}$ and let $f \in \mathfrak{a}, f \neq 0$. After multiplication by a unit, we may assume $f \in \widehat{P}_{n+1}$; by Lemma 5.16 we may also assume $f$ is $y$-regular. Consider the ideal $\mathfrak{a}^{\prime}:=\mathfrak{a} \cap Q_{n}\left[y_{n+1}\right]$. Since $Q_{n}$ is Noetherian, so is $Q_{n}\left[y_{n+1}\right]$, and thus there exist $f_{1}, \ldots, f_{r} \in Q_{n}\left[y_{n+1}\right]$ that generate $\mathfrak{a}^{\prime}$. We claim that $\mathfrak{a}=\left(f, f_{1}, \ldots, f_{r}\right) Q_{n+1}$.

Let $g \in \mathfrak{a}$. By Theorem 5.15, there exist a unit $u \in Q_{n+1}, q \in \widehat{P}_{n+1}$ and $r \in \widehat{P}_{n}\left[y_{n+1}\right]$ such that $u g=q f+r$. Since $r \in \mathfrak{a}^{\prime}$, we can find $v_{1}, \ldots, v_{r} \in Q_{n}$ such that $r=\sum_{j=1}^{r} v_{j} f_{j}$. Hence we have $g=u^{-1} q f+\sum_{j=1}^{r} u^{-1} v_{j} f_{j}$, which proves our claim. 


\section{Embedding codimension}

Let $(A, \mathfrak{m}, k)$ be a local ring. The inclusion of $\mathrm{m} / \mathrm{m}^{2}$ in the graded $\operatorname{ring} \operatorname{gr}(A)$ induces a natural surjective homomorphism of $k$-algebras

$$
\gamma: \operatorname{Sym}_{k}\left(\mathrm{~m} / \mathrm{m}^{2}\right) \rightarrow \operatorname{gr}(A)
$$

Definition 6.1. The embedding codimension of $(A, \mathfrak{m}, k)$ is defined to be

$$
\operatorname{ecodim}(A):=\operatorname{ht}(\operatorname{ker}(\gamma))
$$

Proposition 6.2. For any local ring $(A, \mathfrak{m}, k)$, we have

$$
\operatorname{edim}(A)=\operatorname{dim}(\operatorname{gr}(A))+\operatorname{ecodim}(A) .
$$

In particular, if $A$ is Noetherian then $\operatorname{edim}(A)=\operatorname{dim}(A)+\operatorname{ecodim}(A)$.

Proof. This follows from the fact that for every polynomial ring $P=k\left[x_{i} \mid i \in I\right]$ and every ideal $\mathfrak{a} \subset P$, we have $\operatorname{dim}(P)=\operatorname{dim}(P / \mathfrak{a})+\operatorname{ht}(\mathfrak{a})$ (compare Remark 5.12). For the last assertion we use the fact that $\operatorname{dim}(\operatorname{gr}(A))=\operatorname{dim}(A)$ if $A$ is Noetherian.

Remark 6.3. The formula in Proposition 6.2 is still valid, and informative, when some of the quantities involved are infinite.

Remark 6.4. Higher-rank valuation rings provide examples of finite-dimensional non-Noetherian rings whose embedding dimension is smaller than their dimension. For example, let $A \subset$ $k(x, y)$ be the valuation ring associated to the valuation $v: k(x, y)^{*} \rightarrow \mathbb{Z}_{\text {lex }}^{2}$ given by $v(f)=$ $\left(\operatorname{ord}_{x}(f), \operatorname{ord}_{y}\left(\left.f x^{-\operatorname{ord}_{x}(f)}\right|_{x=0}\right)\right)$. This is a 2-dimensional ring whose maximal ideal is principal, which implies that the embedding dimension is 1 . In particular, the second equation in Proposition 6.2 does not hold for such rings.

Remark 6.5. The embedding codimension of a local ring was studied in the Noetherian setting in [35] under the name regularity defect. One of the main results proved there is that if $\mathfrak{p}$ is a prime ideal of a Noetherian local ring $(A, \mathfrak{m})$ such that $\operatorname{dim}(A)=\operatorname{dim}(A / \mathfrak{p})+\operatorname{dim}\left(A_{\mathfrak{p}}\right)$, then $\operatorname{ecodim}\left(A_{\mathfrak{p}}\right) \leq \operatorname{ecodim}(A)$ [35, Theorem 3]. It would be interesting to find suitable conditions for the same property to hold in the non-Noetherian setting.

We now come to the main result of this section, which gives bounds for the embedding codimension of $A$ from maps into $A$.

Proposition 6.6. Let $\varphi:\left(B, \mathfrak{n}, k_{0}\right) \rightarrow(A, \mathfrak{m}, k)$ be a homomorphism of local rings, and assume that $(B, \mathfrak{n})$ has finite embedding dimension. Let $\varphi^{*}: \mathfrak{n} / \mathfrak{n}^{2} \otimes_{k_{0}} k \rightarrow \mathfrak{m} / \mathrm{m}^{2}$ be the induced $k$-linear map on the Zariski cotangent spaces. Then

$$
\operatorname{ecodim}(A) \geq \operatorname{rank}\left(\varphi^{*}\right)-\operatorname{dim}(\operatorname{gr}(B)) .
$$

In particular, if $B$ is Noetherian then $\operatorname{ecodim}(A) \geq \operatorname{rank}\left(\varphi^{*}\right)-\operatorname{dim}(B)$.

Remark 6.7. A stronger form of Proposition 6.6 is obtained by replacing $\operatorname{dim}(\operatorname{gr}(B))$ with $\operatorname{dim}(\operatorname{gr}(B / \operatorname{ker}(\operatorname{gr}(\varphi))))$ in the displayed formula. Note, in fact, that this sharper form of the proposition follows from the special case of the proposition where $\varphi$ is assumed to be injective.

Remark 6.8. Consider the special case where $\varphi$ is a homomorphism of local $k$-algebras with residue fields $k$ (that is, such that the natural maps $k \rightarrow B / \mathfrak{n}$ and $k \rightarrow A / \mathrm{m}$ are isomorphisms) and with $B$ essentially of finite type. The geometric interpretation is the following. Let $f: X \rightarrow Y$ be a morphism 
of schemes over $k$, with $Y$ of finite type over $k$, and let $p \in X(k)$ and $q=f(p) \in Y(k)$. Denote by $T_{p} f: T_{p} X \rightarrow T_{q} Y$ the map induced on Zariski tangent spaces. Then the proposition gives

$$
\operatorname{ecodim}\left(\mathcal{O}_{X, p}\right) \geq \operatorname{dim}\left(\operatorname{Im}\left(T_{p} f\right)\right)-\operatorname{dim}_{q}(\operatorname{Im}(f)),
$$

where $\operatorname{Im}(f) \subset Y$ is the scheme-theoretic image of $f$. Note in particular that if $X$ is Noetherian, then this formula reduces to the intuitive statement that

$$
\operatorname{dim}\left(T_{p} X\right)-\operatorname{dim}_{p}(X) \geq \operatorname{dim}\left(\operatorname{Im}\left(T_{p} f\right)\right)-\operatorname{dim}_{q}(\operatorname{Im}(f)) .
$$

Another special case is when $f$ is a submersion onto $Y$, in which case the formula reduces to the inequality $\operatorname{ecodim}\left(\mathcal{O}_{X, p}\right) \geq \operatorname{ecodim}\left(\mathcal{O}_{Y, q}\right)$.

Proof of Proposition 6.6. We have the commutative diagram

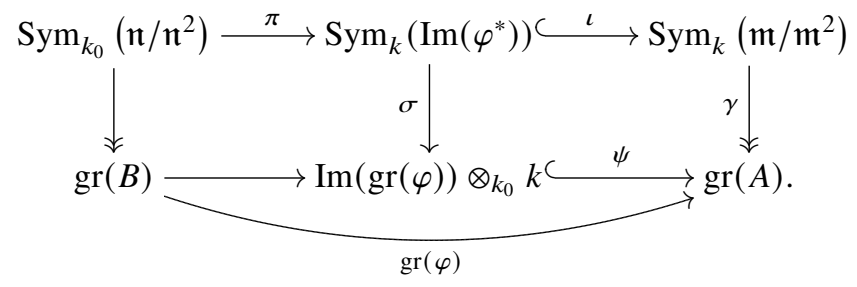

The existence of $\sigma$ follows from the fact that $\operatorname{Im}(\pi) \otimes_{k_{0}} k=\operatorname{Sym}_{k}\left(\operatorname{Im}\left(\varphi^{*}\right)\right)$. The map $\iota$ is a linear extension of polynomial rings, and hence is faithfully flat. Since $\iota^{-1}(\operatorname{ker}(\gamma))=\operatorname{ker}(\sigma)$, we see that

$$
\operatorname{ht}(\operatorname{ker}(\gamma)) \geq \operatorname{ht}(\operatorname{ker}(\sigma))
$$

by the going-down theorem. On the other hand,

$$
\operatorname{ht}(\operatorname{ker}(\sigma))=\operatorname{rank}\left(\varphi^{*}\right)-\operatorname{dim}(\operatorname{Im}(\sigma)) .
$$

Since the inclusion $\operatorname{Im}(\sigma) \subset \operatorname{Im}(\operatorname{gr}(\varphi)) \otimes_{k_{0}} k$ is an inclusion of Noetherian local rings with the same residue field, and $\operatorname{Im}(\operatorname{gr}(\varphi))$ is a quotient of $\operatorname{gr}(B)$, we have

$$
\operatorname{dim}(\operatorname{Im}(\sigma)) \leq \operatorname{dim}(\operatorname{gr}(B)) .
$$

Combining these formulas, we get

$$
\operatorname{ht}(\operatorname{ker}(\gamma)) \geq \operatorname{rank}\left(\varphi^{*}\right)-\operatorname{dim}(\operatorname{gr}(B)) .
$$

To conclude, notice that $\operatorname{dim}(\operatorname{gr}(B))=\operatorname{dim}(B)$ if $B$ is Noetherian.

The following result shows that the embedding codimension of $A$ is invariant under change of the base field, provided the residue field is already contained in $A$ :

Proposition 6.9. Let $(A, \mathrm{~m}, k)$ be a local $k$-algebra such that the natural map $k \rightarrow A / \mathrm{m}$ is an isomorphism, and let $k \subset k^{\prime}$ be a field extension. Denoting $A^{\prime}:=A \otimes_{k} k^{\prime}$, we have

$$
\operatorname{ecodim}\left(A^{\prime}\right)=\operatorname{ecodim}(A) \text {. }
$$

Proof. First, observe that $A^{\prime}$ is a local $k^{\prime}$-algebra with maximal ideal $\mathrm{m}^{\prime}=\mathrm{m} \otimes_{k} k^{\prime}$. We have $\operatorname{ecodim}(A)=\operatorname{ht}(\operatorname{ker}(\gamma))$, where

$$
\gamma: \operatorname{Sym}_{k}\left(\mathrm{~m} / \mathrm{m}^{2}\right) \rightarrow \operatorname{gr}(A)
$$


is defined, as at the beginning. Since for every $n$ we have $\left(\mathfrak{m}^{\prime}\right)^{n} /\left(\mathfrak{m}^{\prime}\right)^{n+1}=\mathfrak{m}^{n} / \mathfrak{n}^{n+1} \otimes_{k} k^{\prime}$, we see that $\gamma$ induces, by base change, the analogous map

$$
\gamma^{\prime}: \operatorname{Sym}_{k}\left(\mathrm{~m}^{\prime} /\left(\mathrm{m}^{\prime}\right)^{2}\right) \rightarrow \operatorname{gr}\left(A^{\prime}\right) .
$$

The next lemma gives ht $\left(\operatorname{ker}\left(\gamma^{\prime}\right)\right)=\operatorname{ht}(\operatorname{ker}(\gamma))$, and the assertion follows.

Lemma 6.10. Let $P=k\left[x_{i} \mid i \in I\right]$ and $P^{\prime}=P \otimes_{k} k^{\prime}=k^{\prime}\left[x_{i} \mid i \in I\right]$, where $k \subset k^{\prime}$ is a field extension. Then for every ideal $\mathfrak{a} \subset P$ we have $\mathrm{ht}(\mathfrak{a})=\operatorname{ht}\left(\mathfrak{a} P^{\prime}\right)$.

Proof. For short, let $\mathfrak{a}^{\prime}=\mathfrak{a} P^{\prime}$. If $I$ is finite, then the lemma follows from dimension theory. In general, suppose by contradiction that $\operatorname{ht}(\mathfrak{a}) \neq \operatorname{ht}\left(\mathfrak{a}^{\prime}\right)$. Then we can find a finite subset $J \subset I$ such that $\operatorname{ht}\left(\mathfrak{a}_{J}\right) \neq \operatorname{ht}\left(\mathfrak{a}_{J}^{\prime}\right)$ (compare Remark 5.12). Since $\mathfrak{a}_{J}^{\prime}=\mathfrak{a}_{J} P_{J}^{\prime}$, this contradicts the finite-dimensional case.

\section{Formal embedding codimension}

In the case of equicharacteristic local rings, looking at the completion instead of the associated graded provides a different way of defining the embedding codimension. To distinguish the two, we introduce the following terminology:

Definition 7.1. The formal embedding codimension of an equicharacteristic local $\operatorname{ring}(A, \mathfrak{m}, k)$ is defined to be

$$
\operatorname{fcodim}(A):=\inf _{\tau} h t(\operatorname{ker}(\tau))
$$

where the infimum is taken over all choices of formal embeddings $\tau: \widehat{P} \rightarrow \widehat{A}$ (see Definition 3.6).

Proposition 7.2. In this definition, we have $\operatorname{fcodim}(A)=\mathrm{ht}(\operatorname{ker}(\tau))$ for every efficient formal embedding $\tau: \widehat{P} \rightarrow \widehat{A}$.

Proof. Given two formal embeddings $\tau: \widehat{P} \rightarrow \widehat{A}$ and $\tau^{\prime}: \widehat{P}^{\prime} \rightarrow \widehat{A}$ with $\tau$ efficient, by Remark 3.13 there is a surjection $\varphi: \widehat{P}^{\prime} \rightarrow \widehat{P}$ such that $\tau^{\prime}=\tau \circ \varphi$, and hence $h t\left(\operatorname{ker}\left(\tau^{\prime}\right)\right) \geq \operatorname{ht}(\operatorname{ker}(\tau))$.

Remark 7.3. If $A$ is a local $k$-algebra such that the residue field $A / \mathrm{m}$ is separable over $k$, then it follows by [32, Chapter $0_{\mathrm{IV}}$, Corollary 19.5.4] that the following are equivalent:

1. $A$ is formally smooth over $k$.

2. $\operatorname{ecodim}(A)=0$.

3. $\operatorname{fcodim}(A)=0$.

Proposition 7.4. For every equicharacteristic local ring $(A, \mathrm{~m}, k)$, we have

$$
\operatorname{edim}(A) \geq \operatorname{dim}(\widehat{A})+\operatorname{fcodim}(A)
$$

and equality holds if $A$ has finite embedding dimension. In particular, if $A$ is Noetherian then $\operatorname{edim}(A)=$ $\operatorname{dim}(A)+\operatorname{fcodim}(A)$.

Proof. Consider an efficient formal embedding $\tau: \widehat{P}=k\left[\left[x_{i} \mid i \in I\right]\right] \rightarrow \widehat{A}$. Note that $\operatorname{dim}(\widehat{P})=$ $\operatorname{edim}(A)$ by Proposition 3.14. The first formula follows from the simple fact that $\operatorname{dim}(\widehat{P}) \geq \operatorname{ht}(\operatorname{ker}(\tau))+$ $\operatorname{dim}(\widehat{P} / \operatorname{ker}(\tau))$. If $A$ has finite embedding dimension, then the set $I$ is finite, and equality holds in the formula because a power series ring in finitely many variables is catenary of dimension equal to the number of variables. The second formula follows from the first and the fact that $\operatorname{dim}(A)=\operatorname{dim}(\widehat{A})$ if $A$ is Noetherian. 
Corollary 7.5. If $(A, \mathrm{~m}, k)$ is an equicharacteristic local ring of finite embedding dimension, then

$$
\operatorname{ecodim}(A)=\operatorname{fcodim}(A) .
$$

Proof. By Propositions 7.4 and 6.2, it suffices to show that $\operatorname{dim}(\operatorname{gr}(A))=\operatorname{dim}(\widehat{A})$. By Proposition 3.7 , the completion $\widehat{A}$ is the quotient of a power series ring in finitely many variables, and therefore is Noetherian and carries the $\widehat{m}$-adic topology. The result now follows from [36, Theorem 15.7] and the identification $\operatorname{gr}(\widehat{A}) \simeq \operatorname{gr}(A)$.

Proposition 7.6. Let $(A, \mathrm{~m}, k)$ be an equicharacteristic local ring. If $A$ admits a DGK decomposition $\widehat{A} \simeq \widehat{B} \hat{\otimes}_{k} \widehat{P}$, then

$$
\operatorname{ecodim}(A)=\operatorname{fcodim}(A)=\operatorname{ecodim}(B)<\infty .
$$

Proof. Since $B$ is Noetherian, we have $\operatorname{edim}(B)<\infty$. By Corollary 7.5, we have that ecodim $(B)=$ fcodim $(B)$. Now we make use of the fact that $A \simeq k\left[\left[x_{i} \mid i \in I\right]\right] / \mathfrak{a}$ with $\mathfrak{a}$ of finite definition. Note that there exists $J \subset I$ finite such that $\mathfrak{a}$ is the extension of $\mathfrak{a}_{J}:=\mathfrak{a} \cap k\left[\left[x_{j} \mid j \in J\right]\right]$ and $\widehat{B} \simeq k\left[\left[x_{j} \mid j \in J\right]\right] / \mathfrak{a}_{J}$. We may assume that the surjection $\tau_{B}: k\left[\left[x_{j} \mid j \in J\right]\right] \rightarrow \widehat{B}$ is an efficient formal embedding; then so is $\tau_{A}: k\left[\left[x_{i} \mid i \in I\right]\right] \rightarrow \widehat{A}$. By Remark 5.3 and Theorem 5.10, it follows that $\operatorname{fcodim}(B)=\mathrm{fcodim}(A)$. It remains to show that ecodim $(B)=\operatorname{ecodim}(A)$.

To that end, note that $\operatorname{gr}\left(\tau_{A}\right)$ factors through the natural surjection $\operatorname{Sym}_{k}\left(\mathrm{~m} / \mathrm{m}^{2}\right) \rightarrow \operatorname{gr}(A)$, and similarly for $\operatorname{gr}\left(\tau_{B}\right)$. We have the commutative diagram

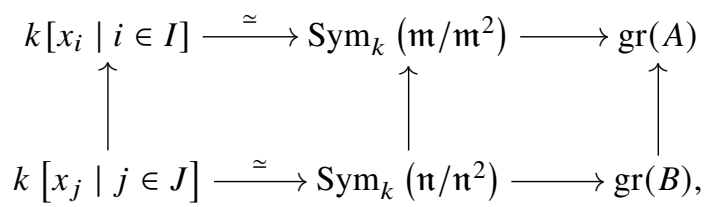

and thus the claim follows by Lemma 5.6.

Remark 7.7. The analogous statement of Proposition 7.6 holds for equicharacteristic local rings $(A, \mathfrak{m}, k)$ admitting a weak DGK decomposition.

The proof of Corollary 7.5 does not extend beyond the case of finite embedding dimension. Nonetheless, the following general comparison theorem holds:

Theorem 7.8. For every equicharacteristic local ring $(A, \mathfrak{m}, k)$, we have

$$
\operatorname{ecodim}(A) \leq \operatorname{fcodim}(A) .
$$

Proof. Fix an efficient formal embedding $\tau: \widehat{P} \rightarrow \widehat{A}$, and let $\operatorname{gr}(\tau): P \rightarrow \operatorname{gr}(A)$ be the induced map on associated graded rings (as in Remark 2.6, we identify $\operatorname{gr}(\widehat{P})=\operatorname{gr}(P)=P$ ). As explained in Remark 3.8, $P \simeq \operatorname{Sym}_{k}\left(\mathrm{~m} / \mathrm{m}^{2}\right)$ and $\operatorname{gr}(\tau)$ gets identified with the canonical surjection $\gamma$. In particular, it is enough to show that

$$
\operatorname{ht}(\operatorname{ker}(\tau)) \geq \operatorname{ht}(\operatorname{ker}(\operatorname{gr}(\tau)))
$$

Write $\mathfrak{a}=\operatorname{ker}(\tau)$. By [7, Chapter III, Section 2.4, Proposition 2], we have $\operatorname{ker}(\operatorname{gr}(\tau))=\operatorname{in}(\mathfrak{a})$. To conclude, it is therefore enough to prove that

$$
\operatorname{ht}(\mathfrak{a}) \geq \operatorname{ht}(\operatorname{in}(\mathfrak{a})) .
$$

This follows from the next proposition. 
Proposition 7.9. Let $P=k\left[x_{i} \mid i \in I\right]$ and $\widehat{P}=k\left[\left[x_{i} \mid i \in I\right]\right]$, where $k$ is a field. Let $\mathfrak{a} \subset \widehat{P}$ be an ideal and $\operatorname{in}(\mathfrak{a}) \subset P$ the corresponding initial ideal. Then $\operatorname{ht}(\mathfrak{a}) \geq \operatorname{ht}(\operatorname{in}(\mathfrak{a}))$.

Proof. If ht $(\operatorname{in}(\mathfrak{a}))<\infty$, then in $(\mathfrak{a})$ is finitely generated and there exists a finite subset $J \subset I$ such that $\operatorname{in}(\mathfrak{a})_{J}$ has the same height as $\operatorname{in}(\mathfrak{a})$ (see Remark 5.12). Otherwise, if $\operatorname{ht}(\operatorname{in}(\mathfrak{a}))=\infty$, then we can pick a finite $J \subset I$ such that the height of in $(\mathfrak{a})_{J}$ is arbitrary large. Define $c:=\operatorname{ht}\left(\operatorname{in}(\mathfrak{a})_{J}\right)$.

By [17, Proposition 1.5.11], we can fix homogeneous elements $g_{1}, \ldots, g_{c} \in \operatorname{in}(\mathfrak{a})_{J}$ forming a regular sequence in $P_{J}$. By the definition of initial ideal, there are elements $f_{1}, \ldots, f_{c} \in \mathfrak{a}$ such that in $\left(f_{i}\right)=g_{i}$ for all $i$. Let $R=R(\widehat{P}):=\bigoplus_{n \in \mathbb{Z}} \widehat{\mathfrak{m}^{n}} u^{-n}$ be the extended Rees algebra of $\widehat{P}$, where we set $\widehat{\mathrm{m}^{n}}=\widehat{P}$ whenever $n<0$. For every $i$, let $\widetilde{f}_{i}:=u^{-\operatorname{ord}\left(f_{i}\right)} f_{i} \in R$. Note that $\left.\widetilde{f}_{i}\right|_{u=0}=\operatorname{in}\left(f_{i}\right)=g_{i}$ via the identification $R / u R \simeq P$.

We claim that for every $1 \leq r \leq c$, the elements $\widetilde{f}_{1}, \ldots, \widetilde{f}_{r}$ form a regular sequence in $R$ and $R /\left(\widetilde{f}_{1}, \ldots, \widetilde{f}_{r}\right)$ is flat over $k[u]$. We argue by induction on $r$, the assertion being clear if $r=0$. Setting for short $B:=R /\left(\widetilde{f}_{1}, \ldots, \widetilde{f}_{r-1}\right)$, we know by induction that $B$ is flat over $k[u]$. Assume that there exists $h \in \widehat{P}$ with $h=\sum_{i=1}^{r-1} a_{i} f_{i}$ and in $(h)$ not divisible by $g_{1}, \ldots, g_{r-1}$. Writing

$$
\sum_{i=1}^{r-1} u^{\operatorname{ord}\left(f_{i}\right)} a_{i} \widetilde{f_{i}}=u^{\operatorname{ord}(h)} \widetilde{h},
$$

Lemma 7.10 yields that $B$ has torsion over $k[u]$, which gives a contradiction. Thus $\operatorname{in}\left(f_{1}, \ldots, f_{r-1}\right)=$ $\left(g_{1}, \ldots, g_{r-1}\right)$ and $B$ is isomorphic to the algebra $\bigoplus_{n \in \mathbb{Z}} \mathfrak{b}_{n} u^{-n}$, where $\mathfrak{b}_{n}:=\widehat{\left(\mathfrak{m}^{n}\right.}+$ $\left.\left(f_{1}, \ldots, f_{r-1}\right)\right) /\left(f_{1}, \ldots, f_{r-1}\right)$ for $n \geq 0$ and $\mathfrak{b}_{n}=B$ for $n<0$. It follows by Proposition 2.7 that $\bigcap_{n \geq 1} \mathfrak{b}_{n}=\{0\}$. Then Lemma 7.11 implies that $B$ is $(u)$-adically separated, and Lemma 7.12 (with $t=u$ ) implies that the class $b$ of $\widetilde{f}_{r}$ in $B$ is a regular element and $B / b B$ is flat over $k[u]$.

The natural isomorphism $R /(u-1) R \simeq \widehat{P}$ sends $\widetilde{f}_{i}$ to $f_{i}$, and hence we see by Lemma 7.12 (with $t=u-1)$ that $f_{1}, \ldots, f_{c}$ form a regular sequence in $\widehat{P}$. This implies that $\operatorname{depth}(\mathfrak{a}, \widehat{P}) \geq c$. We conclude using the fact that $h t(\mathfrak{a}) \geq \operatorname{depth}(\mathfrak{a}, \widehat{P})$ (see, for example, [2, Proposition 2.3 and Lemma 3.2]).

Lemma 7.10. Set $f_{1}, \ldots, f_{r} \in \widehat{P}$. If $h \in \widehat{P}$, then $\operatorname{in}(h) \in\left(\operatorname{in}\left(f_{1}\right), \ldots, \operatorname{in}\left(f_{r}\right)\right)$ if and only if there exist elements $b_{1}, \ldots, b_{r}$ in the Rees algebra $R=R(\widehat{P})$ such that

$$
\widetilde{h}=\sum_{i}^{r} b_{i} \widetilde{f_{i}} .
$$

Proof. Given $\widetilde{h}=\sum b_{i} \widetilde{f}_{i}$, we may assume that $b_{i}$ is homogeneous in $R-$ that is, of the form $b_{i}=$ $u^{-\operatorname{ord}(h)+\operatorname{ord}\left(f_{i}\right)} a_{i}$, with $a_{i} \in \widehat{P}$. But then $\operatorname{ord}\left(a_{i}\right) \geq \operatorname{ord}(h)-\operatorname{ord}\left(f_{i}\right)$ and the claim follows.

Lemma 7.11. Let $A$ be a ring and $\left(\mathfrak{a}_{n}\right)_{n \geq 0}$ a graded sequence of ideals of $A$, and let $R(A):=$ $\bigoplus_{n \in \mathbb{Z}} \mathfrak{a}_{n} u^{-n}$, where we set $\mathfrak{a}_{n}=A$ for $n<0$. Assume that $\bigcap_{n \geq 1} \mathfrak{a}_{n}=\{0\}$. Then $R(A)$ is $(u)$-adically separated.

Proof. Let $a \in R(A)$ be any element. Write $a=\sum_{i=p}^{q} a_{i} u^{-i}$ for some $a_{i} \in \widehat{P}$ and $p, q \in \mathbb{Z}$. By the definition of Rees algebra, we have $a_{i} \in \mathfrak{a}_{i}$ for all $i$. The condition that $a \in u^{n} R(A)$ is equivalent to having $a_{i} \in \mathfrak{a}_{n+i}$ for all $i$. If $a \in \bigcap_{n \geq 1} u^{n} R(A)$, then we have $a_{i} \in \bigcap_{n \geq 1} \mathfrak{a}_{n}=\{0\}$ for all $i$, and hence $a=0$.

Lemma 7.12. Let $B$ be a flat and $k[t]$-algebra. For any given $b \in B$, consider the following properties: 
1. $b$ is a regular element of $B$ and $B / b B$ is flat over $k[t]$.

2. The image $\bar{b}$ of $b$ in $B / t B$ is regular.

Then $(1) \Rightarrow(2)$, and the converse holds if $B$ is $(t)$-adically separated.

Proof. The proof is an adaptation of the proof of [36, Theorem 22.5]. The implication (1) $\Rightarrow(2)$ follows by the snake lemma applied to the commutative diagram

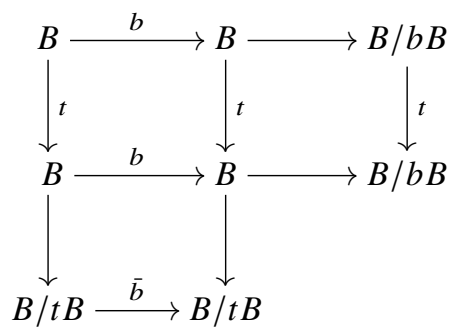

after observing that the map $B \rightarrow B$ given by multiplication by $b$ is injective, since $b$ is regular, and so is the map $B / b B \rightarrow B / b B$ given by multiplication by $t$, since $B / b B$ is flat over $k[t]$.

In order to prove the implication (2) $\Rightarrow(1)$ when $B$ is $(t)$-adically separated, suppose $x \in B$ is an element such that $b x=0$. Then $\bar{b} \bar{x}=0$ in $B / t B$, and hence $\bar{x}=0$. This means that $x \in t B$. Suppose $x \in t^{n} B$ for some positive integer $n$, and write $x=t^{n} y$ in $B$. Then $t^{n}(b y)=b x=0$ and hence $b y=0$, since $B$ is flat over $k[t]$. This implies that $y \in t B$, and hence $x \in t^{n+1} B$. Therefore $x \in \bigcap_{n \geq 1} t^{n} B$, and since $B$ is $(t)$-adically separated, this means that $x=0$. This proves that $b$ is a regular element. To conclude that $B / b B$ is flat over $k[t]$, we just compute $\operatorname{Tor}_{1}^{k[t]}(k, B / b B)=0$ from the exact sequence $0 \rightarrow B \rightarrow B \rightarrow B / b B \rightarrow 0$ and apply [36, Theorem 22.3].

Question 7.13. We do not know of any example where the inequality in Theorem 7.8 is strict. The question whether $\operatorname{ecodim}(A)=\operatorname{fcodim}(A)$ holds for all equicharacteristic local rings $(A, \mathfrak{m}, k)$ is, to our knowledge, still open.

\section{Embedding codimension of arc spaces}

Let $X$ be a scheme of finite type over a field $k$. The arc space $X_{\infty}$ of $X$ is the scheme over $k$ representing the functor of points given, for any $k$-algebra $R$, by $R \mapsto \lim _{m} X_{m}(R)$, where $X_{m}(R)=$ $\operatorname{Hom}_{k}\left(\operatorname{Spec}\left(R[t] /\left(t^{m+1}\right)\right), X\right)$ is the functor of points of the $m$ th jet scheme of $X$. By [5, Remark 4.6], the functor $X_{\infty}(R)$ is naturally isomorphic to $\operatorname{Hom}_{k}(\operatorname{Spec}(R[[t]]), X)$. A point $\alpha \in X_{\infty}$ is called an arc on $X$ and corresponds to a morphism $\operatorname{Spec}(L[[t]]) \rightarrow X$, where $L$ is the residue field of $\alpha$. A point $\alpha \in X_{\infty}$ is said to be constructible if it is the generic point of an irreducible constructible subset of $X_{\infty}$ (compare [21, Section 10]).

Given an $\operatorname{arc} \alpha: \operatorname{Spec}(k[[t]]) \rightarrow X$, we will denote by $\alpha(0)$ and $\alpha(\eta)$ the images in $X$ of the closed point and the generic point of $\operatorname{Spec}(k[[t]])$; we call $\alpha(0)$ the special point of $\alpha$ and $\alpha(\eta)$ the the generic point of $\alpha$.

Given an open set $U \subset X$, we have $\alpha(\eta) \in U$ if and only if the morphism $\alpha: \operatorname{Spec}(k[[t]]) \rightarrow X$ does not factor through the complement $X \backslash U$. We will be interested in the case where $U=X_{\mathrm{sm}}$, the smooth locus of $X$. Note that if $k$ is perfect, then the complement $X \backslash X_{\mathrm{sm}}$ is the singular locus $\operatorname{Sing}(X)$ of $X$.

We following result is a variant of [21, Theorems 9.2 and 9.3]:

Theorem 8.1. Suppose that $X$ is an affine scheme over a perfect field $k$. Let $\alpha \in X_{\infty}$ be an arc and let $d:=\operatorname{dim}_{k(\alpha(\eta))}\left(\Omega_{X / k} \otimes_{k} k(\alpha(\eta))\right)$, where $k(\alpha(\eta))$ is the residue field of $\alpha(\eta) \in X$. Assume that one of the following occurs:

1. $k$ is a field of characteristic 0 or

2. $\alpha \in X_{\infty}(k)$. 
Fix a closed embedding $X \subset \mathbb{A}^{N}$, let $f: X \rightarrow Y:=\mathbb{A}^{d}$ be the morphism induced by a general linear projection $\mathbb{A}^{N} \rightarrow \mathbb{A}^{d}$ and let $\beta:=f_{\infty}(\alpha) \in Y_{\infty}$. Let $\mathrm{m} \subset \mathcal{O}_{X_{\infty}, \alpha}$ and $\mathfrak{n} \subset \mathcal{O}_{Y_{\infty}, \beta}$ be the respective maximal ideals and $L$ and $L^{\prime}$ the residue fields. Then the induced $L$-linear map

$$
\left(T_{\alpha} f_{\infty}\right)^{*}: \mathfrak{n} / \mathfrak{n}^{2} \otimes_{L^{\prime}} L \rightarrow \mathfrak{m} / \mathfrak{m}^{2}
$$

is an isomorphism.

Proof. By assumption, we have that $\operatorname{ord}_{\alpha}\left(\operatorname{Fitt}^{d}\left(\Omega_{X / k}\right)\right)<\infty$, and by taking a general linear projection we can ensure that $\operatorname{ord}_{\alpha}\left(\operatorname{Fitt}^{d}\left(\Omega_{X / k}\right)\right)=\operatorname{ord}_{\alpha}\left(\operatorname{Fitt}^{0}\left(\Omega_{X / Y}\right)\right)$.

Since $k$ is perfect, we have a commutative diagram with exact rows

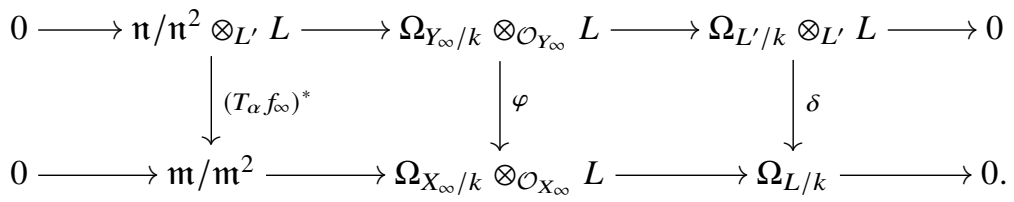

The main step is to understand the map $\varphi$. As in the proof of [21, Theorem 9.2], denote for short $B_{L}:=L[[t]]$ and $P_{L}:=L((t)) / t L[[t]]$.

Note that, by [21, Theorem 5.3], there are natural isomorphisms

$$
\Omega_{X_{\infty} / k} \otimes_{\mathcal{O}_{X_{\infty}}} L \simeq \Omega_{X / k} \otimes_{\mathcal{O}_{X}} P_{L}
$$

and

$$
\Omega_{Y_{\infty} / k} \otimes_{\mathcal{O}_{Y_{\infty}}} L \simeq \Omega_{Y / k} \otimes_{\mathcal{O}_{Y}} P_{L}
$$

We will use these isomorphisms to study $\varphi$.

By pulling back the terms of the exact sequence

$$
\Omega_{Y / k} \otimes_{O_{Y}} \mathcal{O}_{X} \rightarrow \Omega_{X / k} \rightarrow \Omega_{X / Y} \rightarrow 0
$$

along $\alpha$, we obtain the exact sequence

$$
\Omega_{Y / k} \otimes_{\mathcal{O}_{Y}} B_{L} \rightarrow \Omega_{X / k} \otimes_{\mathcal{O}_{X}} B_{L} \rightarrow \Omega_{X / Y} \otimes_{\mathcal{O}_{X}} B_{L} \rightarrow 0 .
$$

Since $Y$ is smooth, we see that the term $F_{Y}:=\Omega_{Y / k} \otimes_{\mathcal{O}_{Y}} B_{L}$ is a free $B_{L}$-module. Write $\Omega_{X / k} \otimes_{\mathcal{O}_{X}} B_{L}=$ $F_{X} \oplus T_{X}$, where $F_{X}$ is free and $T_{X}$ is torsion. Since $\operatorname{ord}_{\alpha}\left(\operatorname{Fitt}^{0}\left(\Omega_{X / Y}\right)\right)<\infty$, the term $T_{X / Y}:=$ $\Omega_{X / Y} \otimes_{\mathcal{O}_{X}} B_{L}$ is a torsion $B_{L}$-module, and we get an exact sequence

$$
0 \rightarrow F_{Y} \rightarrow F_{X} \oplus T_{X} \rightarrow T_{X / Y} \rightarrow 0 .
$$

Since $P_{L}$ is a divisible $B_{L}$-module, tensoring with $P_{L}$ kills torsion, and hence this sequence gives the exact sequence

$$
0 \rightarrow \operatorname{Tor}_{1}^{B_{L}}\left(T_{X}, P_{L}\right) \rightarrow \operatorname{Tor}_{1}^{B_{L}}\left(T_{X / Y}, P_{L}\right) \rightarrow F_{Y} \otimes_{B_{L}} P_{L} \stackrel{\varphi^{\prime}}{\rightarrow} F_{X} \otimes_{B_{L}} P_{L} \rightarrow 0 .
$$

Note that $\varphi^{\prime}=\varphi$ under the aforementioned isomorphisms. We have $\operatorname{Tor}_{1}^{B_{L}}\left(T_{X}, P_{L}\right) \simeq T_{X}$, and this has dimension $\operatorname{ord}_{\alpha}\left(\operatorname{Fitt}^{d}\left(\Omega_{X / k}\right)\right)$ over $L$. Similarly, $\operatorname{Tor}_{1}^{B_{L}}\left(T_{X / Y}, P_{L}\right) \simeq T_{X / Y}$ has dimension $\operatorname{ord}_{\alpha}\left(\operatorname{Fitt}^{0}\left(\Omega_{X / Y}\right)\right)$ over $L$. Since these two dimensions are equal, the map $\varphi^{\prime}$ in the sequence is an isomorphism. We conclude that $\varphi$ is an isomorphism.

The surjectivity of $\varphi$ implies that $\delta$ is surjective, and the injectivity of $\delta$ follows from our assumption that either (1) or (2) holds. We conclude that $\left(T_{\alpha} f_{\infty}\right)^{*}$ is an isomorphism. 
Corollary 8.2. Keeping the assumptions and notation from Theorem 8.1, let $\alpha_{n}, \beta_{n}$ denote the images of $\alpha, \beta$ under the projections $X_{\infty} \rightarrow X_{n}$ and $Y_{\infty} \rightarrow Y_{n}$, and $\mathfrak{m}_{n} \subset \mathcal{O}_{X_{n}, \alpha_{n}}, \mathfrak{n}_{n} \subset \mathcal{O}_{Y_{n}, \beta_{n}}$ denote the corresponding ideals with residue fields $L_{n}, L_{n}^{\prime}$. Then the induced L-linear map

$$
\left(T_{\alpha_{n}} f_{n}\right)^{*}: \mathfrak{n}_{n} / \mathfrak{n}_{n}^{2} \otimes_{L_{n}^{\prime}} L \rightarrow \mathfrak{m}_{n} / \mathrm{m}_{n}^{2} \otimes_{L_{n}} L
$$

is injective for all $n \in \mathbb{N}$.

Proof. This follows from the diagram

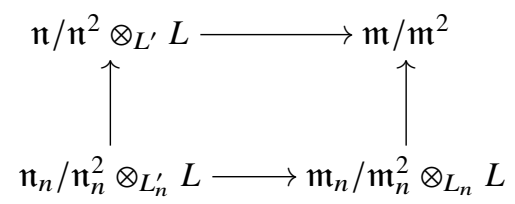

and the fact that the top horizontal and left vertical arrows are injections.

Theorem 8.3. Let $X$ be a scheme of finite type over a perfect field $k$ and let $\alpha \in X_{\infty}$. Assume that one of the following occurs:

1. $k$ is a field of characteristic 0 or

2. $\alpha \in X_{\infty}(k)$.

Then we have

$$
\operatorname{ecodim}\left(\mathcal{O}_{X_{\infty}, \alpha}\right) \leq \limsup _{n \rightarrow \infty} \operatorname{ecodim}\left(\mathcal{O}_{X_{n}, \alpha_{n}}\right)
$$

where $\alpha_{n}$ is the image of $\alpha$ under the truncation map $\pi_{n}: X_{\infty} \rightarrow X_{n}$.

Proof. We can assume without loss of generality that $X$ is affine. Given a map

$$
f: X \rightarrow Y:=\mathbb{A}^{d},
$$

we let $\beta:=f_{\infty}(\alpha) \in Y_{\infty}$. For every $n$, we denote by $\alpha_{n} \in X_{n}$ and $\beta_{n} \in Y_{n}$ the images of $\alpha$ and $\beta$ at the respective $n$-jet schemes. It is convenient, within this proof, to change notation from before to let $A_{\infty}:=\mathcal{O}_{X_{\infty}, \alpha}$ and $B_{\infty}:=\mathcal{O}_{Y_{\infty}, \beta}$, and denote by $\boldsymbol{m}_{\infty} \subset A_{\infty}$ and $\mathfrak{n}_{\infty} \subset B_{\infty}$ the respective maximal ideals and by $L_{\infty}:=A_{\infty} / \mathfrak{m}_{\infty}$ and $L_{\infty}^{\prime}:=B_{\infty} / \mathfrak{n}_{\infty}$ the residue fields. Similarly, for every $n \in \mathbb{N}$, we let $A_{n}:=\mathcal{O}_{X_{n}, \alpha_{n}}$ and $B_{n}:=\mathcal{O}_{Y_{n}, \beta_{n}}$, and denote by $\mathfrak{m}_{n} \subset A_{n}$ and $\mathfrak{n}_{n} \subset B_{n}$ the respective maximal ideals and by $L_{n}:=A_{n} / \mathrm{m}_{n}$ and $L_{n}^{\prime}:=B_{n} / \mathfrak{n}_{n}$ the residue fields.

Note that we have direct systems $\left\{A_{n} \rightarrow A_{n+1} \mid n \in \mathbb{N}\right\}$ and $\left\{B_{n} \subset B_{n+1} \mid n \in \mathbb{N}\right\}$, and $A_{\infty}=\lim _{\longrightarrow} A_{n}$ and $B_{\infty}=\lim _{\longrightarrow} B_{n}$. Moreover, we have commutative diagrams

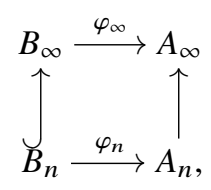

where $\mathfrak{n}_{n}=\varphi_{n}^{-1}\left(\mathrm{~m}_{n}\right), \mathfrak{n}_{n}=\mathfrak{n}_{\infty} \cap B_{n}$ and $\mathfrak{m}_{n}=\mathfrak{m}_{\infty} \cap A_{n}$.

For every $n \in \mathbb{N} \cup\{\infty\}$, let

$$
d \varphi_{n}: \mathfrak{n}_{n} / \mathfrak{n}_{n}^{2} \otimes_{L_{n}^{\prime}} L_{n} \rightarrow \mathfrak{m}_{n} / \mathfrak{m}_{n}^{2}
$$

be the induced $L_{n}$-linear map. 
We pick $f$ as in Theorem 8.1. For every $n \in \mathbb{N} \cup\{\infty\}$, there is an associated map of graded rings $\operatorname{gr}\left(\varphi_{n}\right): \operatorname{gr}\left(B_{n}\right) \rightarrow \operatorname{gr}\left(A_{n}\right)$. We denote by

$$
\psi_{n}: \operatorname{gr}\left(B_{n}\right) \otimes_{L_{n}^{\prime}} L_{\infty} \rightarrow \operatorname{gr}\left(A_{n}\right) \otimes_{L_{n}} L_{\infty}
$$

the map induced by $\operatorname{gr}\left(\varphi_{n}\right)$ by the indicated base changes.

Note that $\mathfrak{m}_{\infty}=\lim _{\longrightarrow} \mathfrak{m}_{n}$ and hence $\mathfrak{m}_{\infty}^{r}=\underline{\lim }_{\longrightarrow} \mathfrak{m}_{n}^{r}$ for all $r$. Indeed, if $a \in \mathfrak{m}_{\infty}^{r}$ for some $r \geq 2$, then we can write $a=\overrightarrow{a_{1}} \cdots a_{r}$ with $a_{i} \in \mathrm{m}_{\infty}$; then we can pick $n$ such that $a_{i} \in \mathfrak{m}_{n}$ for all $i$, and hence $a \in \mathrm{m}_{n}^{r}$. It follows that

$$
\operatorname{gr}\left(A_{\infty}\right)=\lim _{\longrightarrow} \operatorname{gr}\left(A_{n}\right) \otimes_{L_{n}} L_{\infty}
$$

and similarly we have

$$
\operatorname{gr}\left(B_{\infty}\right)=\lim _{\longrightarrow} \operatorname{gr}\left(B_{n}\right) \otimes_{L_{n}^{\prime}} L_{\infty}^{\prime}
$$

Since $\mathfrak{n}_{n}^{r}=\varphi_{n}^{-1}\left(\mathfrak{m}_{n}^{r}\right)$ for all $r$, for every $n$ we have a commutative diagram

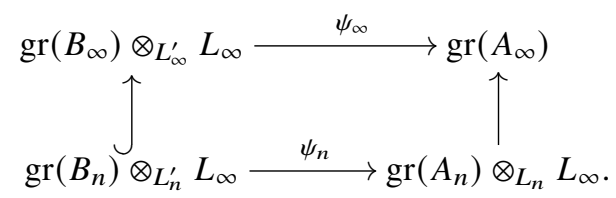

For short, let $R_{n}:=\operatorname{gr}\left(A_{n}\right) \otimes_{L_{n}} L_{\infty}, S_{n}:=\operatorname{gr}\left(B_{n}\right) \otimes_{L_{n}^{\prime}} L_{\infty}$ and $K_{n}:=\operatorname{ker}\left(\psi_{n}\right)$.

Lemma 8.4. $\operatorname{ht}\left(K_{\infty}\right)=\lim \sup _{n} \operatorname{ht}\left(K_{n}\right)$.

Proof. First, note that $K_{\infty}=\lim _{\longrightarrow} K_{n}$. Indeed, the inclusion $K_{\infty} \supset \lim _{\longrightarrow} K_{n}$ is clear, and conversely, if $b \in K_{\infty}$ and we fix $n \in \mathbb{N}$ such that $b \in S_{n}$, then $\psi_{m}(b)$ is in the kernel of $R_{m} \rightarrow R_{\infty}$ for all $m \geq n$ and hence, since each $\psi_{m}(b)$ maps to $\psi_{m+1}(b)$ via $R_{m} \rightarrow R_{m+1}$, it follows that $\psi_{m}(b)$ is zero for $m \gg n$, which means that $b \in K_{m}$ for $m \gg n$.

We are now ready to prove that

$$
\operatorname{ht}\left(K_{\infty}\right)=\underset{n}{\lim \operatorname{supht}}\left(K_{n}\right) .
$$

Note that the maps $S_{n} \rightarrow S_{\infty}$ are extensions of polynomial rings over the same field $L_{\infty}$. Thus they are faithfully flat, and hence for every prime $\mathfrak{p}_{n} \subset S_{n}$ its extension $\mathfrak{p}_{n} S_{\infty}$ is prime.

Consider first the case where $\operatorname{ht}\left(K_{\infty}\right)<\infty$ and let $\mathfrak{p} \subset S_{\infty}$ be a minimal prime over $K_{\infty}$ with $\operatorname{ht}(\mathfrak{p})=\operatorname{ht}\left(K_{\infty}\right)$. By Remark 5.12 we have that $\mathfrak{p}$ is finitely generated by elements $f_{1}, \ldots, f_{r} \in S_{\infty}$. For each $n>0$, let $\mathfrak{p}_{n}$ be any minimal prime over $K_{n}$ contained in $\mathfrak{p} \cap S_{n}$. Then $\mathfrak{p}^{\prime}:=\lim _{\longrightarrow} \mathfrak{p}_{n}$ is a prime of $S_{\infty}$ with $K_{\infty} \subset \mathfrak{p}^{\prime} \subset \mathfrak{p}$, so $\mathfrak{p}^{\prime}=\mathfrak{p}$. Let $n_{1}>0$ be such that $f_{1}, \ldots, f_{r} \in \mathfrak{p}_{n_{1}}$; then $\mathfrak{p}_{n} \vec{a} \cap n S_{n}$ for $n \geq n_{1}$. Given any chain of primes

$$
(0)=\mathfrak{q}_{0} \subsetneq \mathfrak{q}_{1} \subsetneq \cdots \subsetneq \mathfrak{q}_{t}=\mathfrak{p} \subset S_{\infty}
$$

pick $s_{i} \in \mathfrak{q}_{i} \backslash \mathfrak{q}_{i-1}$ and fix $n_{2}$ such that $s_{1}, \ldots, s_{t} \in S_{n_{2}}$. Then for every $n \geq \max \left\{n_{1}, n_{2}\right\}$ we get a chain of primes

$$
(0)=\mathfrak{q}_{0} \cap S_{n} \subsetneq \mathfrak{q}_{1} \cap S_{n} \subsetneq \cdots \subsetneq \mathfrak{q}_{t} \cap S_{n}=\mathfrak{p}_{n} .
$$

Thus $\operatorname{ht}\left(K_{\infty}\right) \leq \lim \sup _{n} \operatorname{ht}\left(K_{n}\right)$. The other inequality follows by the going-down theorem applied to $S_{n} \rightarrow S_{\infty}$. 
If $\operatorname{ht}\left(K_{\infty}\right)=\infty$, then, since $\operatorname{ht}\left(K_{\infty}\right) \geq \operatorname{ht}\left(K_{n} S_{\infty}\right)$, a similar argument shows that the sequence $\left\{\mathrm{ht}\left(K_{n}\right)\right\}_{n}$ is unbounded.

We can now finish the proof of the theorem. Since $B_{n}$ is formally smooth, for every $n \in \mathbb{N} \cup\{\infty\}$ the natural map

$$
\operatorname{Sym}_{L_{n}^{\prime}}\left(\mathfrak{n}_{n} / \mathfrak{n}_{n}^{2}\right) \rightarrow \operatorname{gr}\left(B_{n}\right)
$$

is an isomorphism (see Remark 7.3 for the case $n=\infty$ ). Furthermore, the diagram

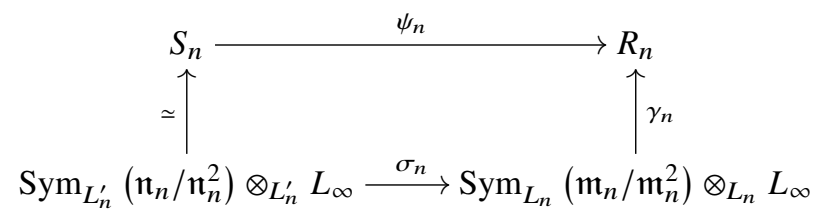

is commutative.

By Theorem 8.1, the map $\sigma_{\infty}$ is an isomorphism, and hence

$$
\operatorname{ht}\left(K_{\infty}\right)=\operatorname{ht}\left(\operatorname{ker}\left(\gamma_{\infty}\right)\right)=\operatorname{ecodim}\left(A_{\infty}\right) .
$$

Similarly, by Corollary $8.2, \sigma_{n}$ is an injective $L_{\infty}$-linear map of polynomial rings, and we have

$$
\operatorname{ht}\left(K_{n}\right) \leq \operatorname{ht}\left(\operatorname{ker}\left(\gamma_{n}\right)\right)=\operatorname{ecodim}\left(A_{n}\right) .
$$

Then we conclude by Lemma 8.4 .

Theorem 8.5. Let $X$ be a scheme of finite type over a perfect field $k$ and let $\alpha \in X_{\infty}$. Assume that either $k$ is a field of characteristic 0 or $\alpha$ is a $k$-rational point. Then we have

$$
\operatorname{ecodim}\left(\mathcal{O}_{X_{\infty}, \alpha}\right) \leq \operatorname{ord}_{\alpha}\left(\operatorname{Fitt}^{d}\left(\Omega_{X / k}\right)\right)
$$

where $d=\operatorname{dim}_{\alpha(\eta)}(X)$. In particular:

1. If $X$ is a variety, then ecodim $\left(\mathcal{O}_{X_{\infty}, \alpha}\right) \leq \operatorname{ord}_{\alpha}\left(\operatorname{Jac}_{X}\right)$.

2. If $\alpha(\eta) \in X_{\mathrm{sm}}$ and $X^{0} \subset X$ is the irreducible component containing $\alpha(\eta)$, then ecodim $\left(\mathcal{O}_{X_{\infty}, \alpha}\right) \leq$ $\operatorname{ord}_{\alpha}\left(\operatorname{Jac}_{X^{0}}\right)<\infty$.

Proof. First note that it suffices to prove the theorem when $\alpha(\eta) \in X_{\mathrm{sm}}$, as otherwise the right-hand side of the stated inequality is infinite and the statement is trivial. Let us therefore assume that $\alpha(\eta) \in X_{\mathrm{sm}}$.

For every $r$, let $J_{r}:=\operatorname{Fitt}^{r}\left(\Omega_{X / k}\right) \subset \mathcal{O}_{X}$. On the one hand, for every finite $n$ we have by [21, Lemma 8.1]

$$
\operatorname{edim}\left(\mathcal{O}_{X_{n}, \alpha_{n}}\right)=(n+1) d_{n}-\operatorname{dim}\left(\overline{\left\{\alpha_{n}\right\}}\right)+\operatorname{ord}_{\alpha}\left(J_{d_{n}}\right)
$$

where $d_{n}=d\left(\alpha_{n}, \Omega_{X / k}\right)$ is the Betti number of $\Omega_{X / k}$ with respect to $\alpha_{n}$ [21, Definition 6.1] and $\overline{\left\{\alpha_{n}\right\}}$ the closure of $\alpha_{n}$ in $X_{n}$. On the other hand, since $\alpha$ is not in an irreducible component of $X_{\infty}$ that is fully contained in $(\operatorname{Sing}(X))_{\infty}$, we have

$$
\operatorname{dim}\left(\mathcal{O}_{X_{n}, \alpha_{n}}\right) \geq(n+1) d-\operatorname{dim}\left(\overline{\left\{\alpha_{n}\right\}}\right)
$$

for all finite $n$. Since for all $n$ large enough we have $d_{n}=d$, we deduce by Proposition 6.2 that $\operatorname{ecodim}\left(\mathcal{O}_{X_{n}, \alpha_{n}}\right) \leq \operatorname{ord}_{\alpha}\left(J_{d}\right)$ for all $n \gg 1$. We conclude by Theorem 8.3 that $\operatorname{ecodim}\left(\mathcal{O}_{X_{\infty}, \alpha}\right) \leq$ $\operatorname{ord}_{\alpha}\left(J_{d}\right)$, as stated. 
Regarding the last two assertions of the theorem, (1) follows by the fact that if $X$ is a variety, then by definition, $\operatorname{Jac}_{X}=\operatorname{Fitt}^{d}\left(\Omega_{X / k}\right)$. As for (2), if $\alpha(\eta) \in X_{\mathrm{sm}}$, then by Lemma 8.6 we have $\overline{\mathcal{O}_{X_{\infty}, \alpha}} \simeq \overline{\mathcal{O}_{X_{\infty}^{0}, \alpha}}$, and hence we can apply (1) to $X^{0}$; note also that in this case we have $\alpha \in X_{\text {sm }}^{0}$ and hence $\operatorname{ord}_{\alpha}\left(\mathrm{Jac}_{X^{0}}\right)<\infty$.

We include a proof of the following property, which is well known to experts and is remarked in [24]:

Lemma 8.6. Let $X$ be a scheme of finite type over a field $k$ and $\alpha \in X_{\infty}$ an arc with $\alpha(\eta) \in X_{\mathrm{sm}}$. Let $X^{0} \subset X$ be the irreducible component containing $\alpha(\eta)$. Then $\overline{\mathcal{O}_{X_{\infty}, \alpha}} \simeq \overline{\mathcal{O}_{X_{\infty}^{0}, \alpha}}$.

Proof. We may assume that $X=\operatorname{Spec}(R)$ is affine. By abuse of notation, we write $\alpha$ for the map $R \rightarrow A[[t]]$. Let $\mathfrak{a}:=\operatorname{ker}(\alpha)$. If $(0)=\prod_{i} \mathfrak{q}_{i} \subset R$ is a primary decomposition with $\mathfrak{q}_{0}$ the minimal prime defining $X^{0}$, then the condition $\alpha(\eta) \in X^{0}$ translates to $\mathfrak{q}_{0} \subset \mathfrak{a}$ and $\mathfrak{q}_{i} \not \subset \mathfrak{a}$ for $i \neq 0$. Let $A$ be a test-ring - that is, it is local with maximal ideal $\mathrm{m}$, residue field $K$ equal to the residue field of $\alpha \in X_{\infty}$ and $\mathrm{m}^{n}=0$ for some $n \in \mathbb{N}$. Let $\alpha^{\prime}$ be any $A$-deformation of $\alpha-$ that is, given by a map $R \rightarrow A[[t]]$. To prove the lemma, it suffices to show that $\mathfrak{a}^{\prime}:=\operatorname{ker}\left(\alpha^{\prime}\right) \supset \mathfrak{q}_{0}$. We have the commutative diagram

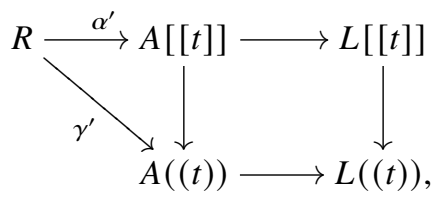

where $A((t))$ denotes the localisation of $A[[t]]$ at the ideal $\mathrm{m}$. Since $A[[t]] \rightarrow A((t))$ is injective, we have $\mathfrak{a}^{\prime}=\operatorname{ker}\left(\gamma^{\prime}\right)$. Let $f \in \mathfrak{q}_{0}$. Take any $f_{i} \in \mathfrak{q}_{i} \backslash \mathfrak{a}$ for $i \neq 0$. Then $g:=f \prod_{i} f_{i} \in \mathfrak{a}^{\prime}$. Since $\gamma^{\prime}\left(f_{i}\right) \neq 0$ modulo $\mathrm{m}$, we have that $\gamma^{\prime}\left(f_{i}\right)$ is a unit. Thus $0=\gamma^{\prime}(g)=\gamma^{\prime}(f) u$, where $u$ is a unit, and in particular $f \in \mathfrak{a}^{\prime}$.

Theorem 8.7. Let $X$ be a scheme of finite type over a perfect field $k$. For $\alpha \in X_{\infty}$ such that $\alpha(\eta) \in X \backslash X_{\mathrm{sm}}$, we have ecodim $\left(\mathcal{O}_{X_{\infty}, \alpha}\right)=\infty$.

Proof. Note that since $k$ is perfect, we have $X \backslash X_{\mathrm{sm}}=\operatorname{Sing}(X)$, and in particular the condition that $\alpha(\eta) \in X \backslash X_{\mathrm{sm}}$ is equivalent to having $\alpha \in(\operatorname{Sing}(X))_{\infty}$.

For every $n \in \mathbb{N}$, let $\pi_{n}: X_{\infty} \rightarrow X_{n}$ be the truncation morphism, and let $\alpha_{n}:=\pi_{n}(\alpha) \in X_{n}$. Note that for $n=0$ we have $\alpha_{0}=\alpha(0)$. Let $L$ and $L_{n}$ denote the residue fields of $X_{\infty}$ at $\alpha$ and of $X_{n}$ at $\alpha_{n}$. By [21, Lemma 8.3] (see also [21, Remark 7.4]), for all $n$ sufficiently large the differential map

$$
\left(T_{\alpha} \pi_{n}\right)^{*}: \mathfrak{m}_{\alpha_{n}} / \mathfrak{m}_{\alpha_{n}}^{2} \otimes_{L_{n}} L \rightarrow \mathfrak{m}_{\alpha} / \mathfrak{m}_{\alpha}^{2}
$$

has rank at least $(n+1) d(\alpha)-\operatorname{dim}\left(\overline{\left\{\alpha_{n}\right\}}\right)$, where

$$
d(\alpha):=\operatorname{dim}_{k(\alpha(\eta))}\left(\Omega_{X / k} \otimes k(\alpha(\eta))\right) .
$$

Then by Proposition 6.6 we have

$$
\begin{aligned}
\operatorname{ecodim}\left(\mathcal{O}_{X_{\infty}, \alpha}\right) & \geq(n+1) d(\alpha)-\operatorname{trdeg}_{k}\left(L_{n}\right)-\operatorname{dim}\left(\mathcal{O}_{\overline{\pi_{n}\left(X_{\infty}\right)}, \alpha_{n}}\right) \\
& =(n+1) d(\alpha)-\operatorname{dim}_{\alpha_{n}}\left(\overline{\pi_{n}\left(X_{\infty}\right)}\right)
\end{aligned}
$$

where $\overline{\pi_{n}\left(X_{\infty}\right)}$ denotes the Zariski closure of $\pi_{n}\left(X_{\infty}\right)$ in $X_{n}$.

Since $X$ is of finite type, $X_{\infty}$ has finitely many irreducible components (see [41, Theorem 2.9] and [39, Corollary 3.16]). This implies that for $n$ sufficiently large, we have

$$
\operatorname{dim}_{\alpha_{n}}\left(\overline{\pi_{n}\left(X_{\infty}\right)}\right)=\max _{C \ni \alpha} \operatorname{dim}\left(\overline{\pi_{n}(C)}\right),
$$

where the maximum is taken over the irreducible components $C$ of $X_{\infty}$ that contain $\alpha$. 
Let $C$ be one of the irreducible components of $X_{\infty}$ containing $\alpha$, let $\beta \in C$ be its generic point and let $Z \subset X$ be the closure of $\beta(\eta)$ in $X$. From [21, Lemma 8.6], we have

$$
\operatorname{dim}\left(\overline{\pi_{n}(C)}\right) \leq(n+1) \operatorname{dim}(Z) \leq(n+1) \operatorname{dim}_{\alpha(0)}(X)
$$

Since $\alpha(\eta) \in \operatorname{Sing}(X)$, we see by the definition of $d(\alpha)$ that $d(\alpha)>\operatorname{dim}_{\alpha(0)}(X)$, and therefore

$$
\lim _{n \rightarrow \infty}\left((n+1) d(\alpha)-\operatorname{dim}\left(\overline{\pi_{n}(C)}\right)\right) \geq \lim _{n \rightarrow \infty}(n+1)\left(d(\alpha)-\operatorname{dim}_{\alpha(0)}(X)\right)=\infty .
$$

We conclude that ecodim $\left(\mathcal{O}_{X_{\infty}, \alpha}\right)=\infty$, as claimed.

Corollary 8.8. Let $X$ be a scheme of finite type over a field $k$ and let $\alpha \in X_{\infty}$. Assume that either $k$ has characteristic 0 or $\alpha \in X_{\infty}(k)$. Then we have $\alpha(\eta) \in X_{\mathrm{sm}}$ if and only if $\operatorname{ecodim}\left(\mathcal{O}_{X_{\infty}, \alpha}\right)<\infty$.

Proof. If $k$ has characteristic 0 , then the corollary follows by Theorems 8.5 and 8.7 .

Let then $k$ be any field, and assume that $\alpha \in X_{\infty}(k)$. For a field extension $k \subset k^{\prime}$, we denote $X^{\prime}:=X \times_{\operatorname{Spec}(k)} \operatorname{Spec}\left(k^{\prime}\right)$ and let $\alpha^{\prime}: \operatorname{Spec}\left(k^{\prime}[[t]]\right) \rightarrow X^{\prime}$ be the arc obtained by base change from $\alpha$. Since a point of $X$ is in the smooth locus if and only if it is geometrically regular, we can find a field extension $k \subset k^{\prime}$ such that $\alpha^{\prime}$ is not a regular point of $X^{\prime}$. By faithfully flat descent of regularity, we can replace $k^{\prime}$ with a larger field extension and assume without loss of generality that $k^{\prime}$ is perfect. Note that $X_{\infty}^{\prime} \simeq X_{\infty} \times_{\operatorname{Spec}(k)} \operatorname{Spec}\left(k^{\prime}\right)$, and hence $\mathcal{O}_{X_{\infty}^{\prime}, \alpha^{\prime}} \simeq \mathcal{O}_{X_{\infty}, \alpha} \otimes_{k} k^{\prime}$. Then by Proposition 6.9 we have

$$
\operatorname{ecodim}\left(\mathcal{O}_{X_{\infty}^{\prime}, \alpha^{\prime}}\right)=\operatorname{ecodim}\left(\mathcal{O}_{X_{\infty}, \alpha}\right)
$$

This reduces to the case of perfect fields, where the result follows again by Theorems 8.5 and 8.7.

\section{On Drinfeld, Grinberg and Kazhdan's theorem}

Theorem 8.5 can be seen as a finiteness statement for singularities of the arc space at arcs that are not fully contained in the singular locus. One of the first major results in this direction is the theorem of Drinfeld, Grinberg and Kazhdan, which we will state here in its version from [24]. Recall that for any equicharacteristic local ring $(A, \mathfrak{m}, k)$, a DGK decomposition is an isomorphism $\widehat{A} \simeq k\left[\left[t_{i} \mid i \in I\right]\right] / \mathfrak{a}$, where $\mathfrak{a}$ is an ideal of finite polynomial definition.

Theorem 9.1 ([31, Theorem 2.1], [24, Theorem 0.1]). Let $X$ be a scheme of finite type over a field $k$, and let $\alpha \in X_{\infty}(k)$. If $\alpha(\eta) \in X_{\mathrm{sm}}$, then the local ring $\mathcal{O}_{X_{\infty}, \alpha}$ admits a DGK decomposition.

As mentioned in Remark 5.3, any DGK decomposition of $\mathcal{O}_{X_{\infty}, \alpha}$ induces an isomorphism of formal schemes

$$
\widehat{X_{\infty, \alpha}} \simeq \widehat{Z_{z}} \hat{\times} \Delta^{\mathbb{N}}
$$

with $Z$ a scheme of finite type over $k, z \in Z(k)$ and $\Delta=\operatorname{Spf}(k[[t]])$. The formal scheme $\widehat{Z_{z}}$ is often referred to as a formal model for $\alpha$. While $\widehat{Z}_{z}$ is not unique, there exists a unique minimal one in the following sense:

Theorem 9.2 ([10, Theorem 7.1], [12, Theorem 1.2]). Let $\widehat{Z_{z}}$ and $\widehat{W_{w}}$ be two formal models for $\alpha$ which are indecomposable - that is, they are not of the form $\mathcal{Y} \hat{x} \Delta$, with $\mathcal{Y}$ a formal scheme. Then $\widehat{Z_{z}} \simeq \widehat{W_{w}}$.

Definition 9.3. The indecomposable formal model of $\alpha$ is called the minimal formal model and denoted by $\mathcal{Z}_{\alpha}^{\mathrm{min}}$.

Note that the formal model provided by Theorem 9.1 is not minimal in general, which we will see in Section 10. 
Combining Theorem 9.1 with the results of this paper, we obtain the next result, which provides a characterisation of $k$-rational arcs admitting a DGK decomposition. The result also gives an explicit bound for the embedding codimension; we should stress that such a bound does not follow from the proofs in [31, 24, 25].

Theorem 9.4. Let $X$ be a scheme of finite type over a field $k$. For any $\alpha \in X_{\infty}(k)$, the following are equivalent:

1. $\alpha(\eta) \in X_{\mathrm{sm}}$.

2. $\mathcal{O}_{X_{\infty}, \alpha}$ admits a DGK decomposition.

3. $\mathcal{O}_{X_{\infty}, \alpha}$ admits a weak DGK decomposition.

4. $\operatorname{ecodim}\left(\mathcal{O}_{X_{\infty}, \alpha}\right)<\infty$.

Moreover, if $k$ is perfect and $\alpha(\eta) \in X_{\mathrm{sm}}$, then

$$
\operatorname{ecodim}\left(\mathcal{O}_{X_{\infty}, \alpha}\right) \leq \operatorname{ord}_{\alpha}\left(\operatorname{Jac}_{X^{0}}\right)
$$

where $X^{0} \subset X$ is the irreducible component containing $\alpha(\eta)$.

Proof. The implication (1) $\Rightarrow(2)$ is Theorem 9.1, the implication (2) $\Rightarrow$ (3) is obvious, the implication (3) $\Rightarrow$ (4) follows from Corollary 5.11 and finally Corollary 8.8 gives the implication (4) $\Rightarrow$ (1). The last statement follows from Theorem 8.5.

Example 9.5. Let $X$ be the hypersurface defined by $x_{0} x_{n+1}+f\left(x_{1}, \ldots, x_{n}\right)=0$ and $\alpha \in X(k)$ the arc given by $(t, 0, \ldots, 0) \in k[[t]]^{n+2}$. Assume further that the hypersurface $H \subset \mathbb{A}^{n}$ given by $f\left(x_{1}, \ldots, x_{n}\right)=0$ has a singularity at 0 . Then, as shown in [24], a DGK decomposition for $\mathcal{O}_{X_{\infty}, \alpha}$ is given by

$$
\widehat{\mathcal{O}_{X_{\infty}, \alpha}} \simeq k\left[\left[x_{1}, \ldots, x_{n}\right]\right] / f\left(x_{1}, \ldots, x_{n}\right) \hat{\otimes}_{k} k\left[\left[t_{i} \mid i \in \mathbb{N}\right]\right]
$$

The singularity of $\alpha$ is thus again given by $H$ and ecodim $\left(\mathcal{O}_{X_{\infty}, \alpha}\right)=1$. On the other hand, the order of $\alpha$ with respect to the $\mathrm{Jacobian}_{\text {ideal }} \mathrm{Jac}_{X}$ is 1 , and hence the bound in Theorem 9.4 is sharp in this case.

Example 9.6. Similar to the previous example, let $X$ be the hypersurface defined by $x_{0} x_{n+1}+$ $f\left(x_{1}, \ldots, x_{n}\right)=0$, where $f$ is a polynomial of multiplicity 2 , and take this time $\alpha \in X_{\infty}(k)$ to be the arc given by $\left(t^{m}, 0, \ldots, 0\right) \in k[[t]]^{n+2}$. Denoting by $g^{(j)}$ the $j$ th Hasse-Schmidt derivative of an element $g \in k\left[x_{0}, \ldots, x_{n+1}\right]$ and setting for short $I=\{0,1, \ldots, n+1\}$ and $J=\mathbb{Z}_{\geq 0}, X_{\infty}$ is defined by the ideal $\mathfrak{a}=\left(\left(x_{0} x_{n+1}+f\right)^{(j)} \mid j \in J\right)$ of $P:=k\left[x_{i}^{(j)} \mid(i, j) \in I \times J\right]$ [45]. Let $\mathfrak{m} \subset P$ be the maximal ideal at $\alpha$. Since $x_{0}^{(m)}$ is a unit in the local ring $P_{\mathfrak{m}}$, we see that the ideal $\operatorname{in}\left(\mathfrak{a} P_{\mathfrak{m}}\right)$ is generated by the elements $x_{n+1}^{(j)}$ for $j \in J$ and in $\left(f^{(l)}\right)$ for $0 \leq l \leq m-1$. As long as $f$ is chosen so that in $\left(f^{(l)}\right)$, $0 \leq l \leq m-1$, form a regular sequence (for example, $f=x_{1} x_{2}$ would work), we get that $\mathcal{O}_{X_{\infty}, \alpha}$ has embedding codimension $m$. Since clearly the order of $\alpha$ with respect to $\mathrm{Jac}_{X}$ is also $m$, this shows that the bound in Theorem 9.4 is sharp for all possible orders of the arc with the Jacobian ideal of $X$.

Let us mention here the following consequence of Theorem 9.4, which implies that the local rings of closed arcs in the arc space provide plenty of examples of non-Noetherian rings whose embedding codimension agrees with their formal embedding codimension.

Corollary 9.7. If $X$ is a scheme of finite type over a field $k$, then

$$
\operatorname{ecodim}\left(\mathcal{O}_{X_{\infty}, \alpha}\right)=\operatorname{fcodim}\left(\mathcal{O}_{X_{\infty}, \alpha}\right)
$$

for every $\alpha \in X_{\infty}(k)$. If $k$ is perfect, then the same holds for all constructible points $\alpha \in X_{\infty}$ with $\alpha(\eta) \in X_{\mathrm{sm}}$. 
Proof. Assume first that $\alpha \in X_{\infty}(k)$. If $\alpha(\eta) \in X_{\mathrm{sm}}$, then the equality follows by Theorem 9.1 and Proposition 7.6. If $\alpha(\eta) \in X \backslash X_{\mathrm{sm}}$, then we have ecodim $\left(\mathcal{O}_{X_{\infty}, \alpha}\right)=\infty$ by Corollary 8.8, and we conclude by Theorem 7.8. Suppose now that $\alpha \in X_{\infty}$ is a constructible point with $\alpha(\eta) \in X_{\mathrm{sm}}$. By [21, Theorem 10.8], $\mathcal{O}_{X_{\infty}, \alpha}$ has finite embedding dimension, and hence the assertion follows by Corollary 7.5.

We now state the following application of Theorems 8.1 and 9.1, which says that a generic projection of the base scheme induces an efficient DGK decomposition at an arc that is not contained in the singular locus:

Theorem 9.8. Let $X \subset \mathbb{A}^{N}$ be an affine scheme of finite type over a perfect field $k$, let $\alpha \in X_{\infty}(k)$ with $\alpha(\eta) \in X_{\mathrm{sm}}$ and let $d=\operatorname{dim}_{\alpha(\eta)}(X)$. Let $f: X \rightarrow Y:=\mathbb{A}^{d}$ be the map induced by a general linear projection $\mathbb{A}^{N} \rightarrow \mathbb{A}^{d}$, and let $\beta:=f_{\infty}(\alpha)$. Then the associated map

$$
\varphi: \mathcal{O}_{Y_{\infty}, \beta} \rightarrow \mathcal{O}_{X_{\infty}, \alpha}
$$

gives an efficient formal embedding of $\mathcal{O}_{X_{\infty}, \alpha}$. Moreover, if $k$ is infinite, then there exist formal coordinates $u_{i} \in \overline{\mathcal{O}_{Y_{\infty}, \beta}}, i \in \mathbb{N}$, such that $\operatorname{ker}(\widehat{\varphi})$ is generated by finitely many polynomials in $u_{i}$, and hence $\widehat{\varphi}$ induces an efficient DGK decomposition.

Proof. The first part follows from Theorem 8.1, together with the fact that $\mathcal{O}_{Y_{\infty}, \beta}$ is formally smooth over $k$. Regarding the second assertion, we know by Theorem 9.1 that the map $\varphi$ induces a surjection

$$
\psi: \overline{\mathcal{O}_{Y_{\infty}, \beta}} \rightarrow \overline{\mathcal{O}_{Z, z}} \hat{\otimes}_{k} k\left[\left[t_{i} \mid i \in \mathbb{N}\right]\right]
$$

with $Z$ a scheme of finite type over $k$ and $z \in Z(k)$. If $Z$ is smooth at $z$, then there is nothing to show. Otherwise, by Theorem 3.15 we may assume that $Z \subset \mathbb{A}^{n}$, where $n=\operatorname{edim}\left(\mathcal{O}_{Z, z}\right)$. Since $\psi$ induces an isomorphism of continuous cotangent spaces, the statement follows from Proposition 3.9.

The next example illustrates in concrete terms the content of Theorem 9.8 when $X$ is a hypersurface in an affine space, where the existence of the efficient formal embedding as in the theorem can be verified directly from the equations.

Example 9.9. Let $f \in k\left[x_{1}, \ldots, x_{n}, y\right]$ and let $X$ be the hypersurface defined by $f$. For the sake of convenience, we will write $x=\left(x_{1}, \ldots, x_{n}\right)$. Let $\alpha=(x(t), y(t))$ be an $\operatorname{arc}$ on $X$ such that ord $\operatorname{or}_{\alpha}\left(\operatorname{Jac}_{X}\right)=$ $\operatorname{ord}_{t}\left(\frac{\partial f}{\partial y}(x(t), y(t))\right)=d>0$. We write $x(t)=\sum_{j} a^{(j)} t^{j}$ and $y(t)=\sum_{j} b^{(j)} t^{j}$; note that $a^{(j)}=$ $\left(a_{1}^{(j)}, \ldots, a_{n}^{(j)}\right)$. Let $D=\left(D_{p}\right)_{p \geq 0}$ be the universal Hasse-Schmidt derivation on $k\left[x^{(j)}, y^{(j)} \mid j \geq 0\right]$, where $x^{(j)}=\left(x_{1}^{(j)}, \ldots, x_{n}^{(j)}\right)$. Then $X_{\infty}=\operatorname{Spec}\left(R_{\infty}\right)$, where

$$
R_{\infty}=k\left[x^{(j)}, y^{(j)} \mid j \geq 0\right] /\left(f^{(p)} \mid p \geq 0\right),
$$

with $f^{(p)}:=D_{p}(f)$. Note that $f^{(p)}$ depends only on $x^{(j)}, y^{(j)}$ for $j \leq p$. The arc $\alpha$ then corresponds to the ideal $\mathrm{m}_{\alpha}$ of $R_{\infty}$ given by

$$
\mathbf{m}_{\alpha}=\left(x^{(j)}-a^{(j)}, y^{(j)}-b^{(j)} \mid j \geq 0\right) .
$$

Setting $\widetilde{f^{(p)}}\left(x^{(j)}, y^{(j)}\right):=f^{(p)}\left(x^{(j)}+a^{(j)}, y^{(j)}+b^{(j)}\right)$, we get

$$
\mathcal{O}_{X_{\infty}, \alpha} \simeq k\left[x^{(j)}, y^{(j)}\right]_{\left(x^{(j)}, y^{(j)}\right)} /\left(\widetilde{f^{(p)}}\right) .
$$


We are going to make use of the following explicit formula from [21, Section 5]:

$$
\frac{\partial f^{(p)}}{\partial y^{(q)}}=D_{p-q}\left(\frac{\partial f}{\partial y}\right), \quad q \leq p .
$$

The condition $\operatorname{ord}_{t}\left(\frac{\partial f}{\partial y}(x(t), y(t))\right)=d$ implies that, for $p \geq d$,

$$
\frac{\partial \widetilde{f^{(p)}}}{\partial y^{(q)}}(0,0)=\frac{\partial f^{(p)}}{\partial y^{(q)}}(a, b)=D_{p-q}\left(\frac{\partial f}{\partial y}\right)(a, b) \begin{cases}=0, & p-d<q \leq p \\ \neq 0, & q=p-d\end{cases}
$$

This implies that the initial forms of $f^{(p)}$, for $p \geq d$, can be written as

$$
\text { in }\left(\overline{f^{(d+i)}}\right)=y^{(i)}+g^{(d+i)},
$$

where $g^{(d)} \in k\left[x^{(j)} \mid j \leq d\right]$ and, for $i>0, g^{(d+i)} \in k\left[x^{(j)}, y^{(l)} \mid j \leq d+i, l<i\right]$. In particular, the elements $x^{(j)}$ and $\widetilde{f^{(d+j)}}$, for $j \geq 0$, give formal coordinates in $k\left[\left[x^{(j)}, z^{(j)} \mid j \geq 0\right]\right]$, and hence the map

$$
\varphi: k\left[\left[x^{(j)}, z^{(j)} \mid j \geq 0\right]\right] \rightarrow k\left[\left[x^{(j)}, y^{(j)} \mid j \geq 0\right]\right], \quad x^{(j)} \mapsto x^{(j)}, z^{(j)} \mapsto \overline{f^{(j+d)}}
$$

is an isomorphism. Write $h_{i}:=\varphi^{-1}\left(f^{(i)}\right)$ and $\mathfrak{a}:=\left(\bar{h}_{0}, \ldots, \bar{h}_{d-1}\right)$, where $\bar{h}_{i}$ is obtained from $h_{i}$ by setting $z^{(j)}=0$ for all $j \geq 0$. Then we get

$$
\overline{\mathcal{O}_{X_{\infty}, \alpha}} \simeq k\left[\left[x^{(j)} \mid j \geq 0\right]\right] / \widehat{\mathfrak{a}}
$$

Observe that the map $k\left[\left[x^{(j)} \mid j \geq 0\right]\right] \rightarrow \overline{\mathcal{O}_{X_{\infty}, \alpha}}$ is the efficient formal embedding from Theorem 9.8 with respect to the projection $(x, y) \mapsto x$. However, this isomorphism does not induce a DGK decomposition a priori, since the ideal $\widehat{\mathfrak{a}}$ is not necessarily of finite polynomial definition with respect to the variables $x^{(j)}$.

\section{Efficient embedding of the Drinfeld model}

It is useful to compare the formal embedding given by Theorem 9.8 to the one provided by the DrinfeldGrinberg-Kazhdan theorem. This comparison is done in Theorem 10.2. We first need to recall the construction of the Drinfeld models.

Let $X \subset \mathbb{A}^{N}$ be an affine scheme of finite type over a field $k$, consider a $k$-rational arc $\alpha \in X_{\infty}(k)$ such that $\alpha(\eta) \in X_{\text {sm }}$ and let $d:=\operatorname{dim}_{\alpha(\eta)}(X)$ and $c:=N-d$. Let $X^{0}$ be the irreducible component of $X$ containing $\alpha(\eta)$ (note that $d=\operatorname{dim} X^{0}$ ), and let $X^{\prime} \supset X^{0}$ be the complete intersection scheme defined by the vanishing of $c$ general linear combinations $p_{1}, \ldots, p_{c}$ of a set of generators of the ideal of $X^{0}$ in $\mathbb{A}^{N}$. As explained in [24], the respective inclusions induce isomorphisms $\overline{\mathcal{O}_{X_{\infty}, \alpha}} \simeq \overline{\mathcal{O}_{X_{\infty}^{0}, \alpha}} \simeq \overline{\mathcal{O}_{X_{\infty}^{\prime}, \alpha}}$ (detailed proofs are given in Lemma 8.6 and [10, Section 4.2]). Pick coordinates $x_{1}, \ldots, x_{d}, y_{1}, \ldots, y_{c}$ in the ambient affine space $\mathbb{A}^{N}$. For a general choice of such coordinates, we can assume that

$$
\operatorname{ord}_{\alpha}\left(\operatorname{det}\left(\frac{\partial\left(p_{1}, \ldots, p_{c}\right)}{\partial\left(y_{1}, \ldots, y_{c}\right)}\right)\right)=\operatorname{ord}_{\alpha}\left(\operatorname{Jac}_{X^{\prime}}\right)=\operatorname{ord}_{\alpha}\left(\operatorname{Jac}_{X^{0}}\right)=: e<\infty .
$$

Drinfeld defines a specific formal model for $\overline{\mathcal{O}_{X_{\infty}, \alpha}}$ depending only on the choices of the coordinates $x_{i}, y_{j}$, the equations $p_{l}$ and the order of contact $e$. Concretely, consider the affine space $\mathbb{A}^{m}$, where $m=e(1+2 d+c)$. We denote by $R[t]_{<n}$ the space of polynomials of degree $<n$ with coefficients in $R$. Denoting by $Q_{n}$ the scheme representing the functor $R \mapsto t^{n}+R[t]_{<n}$, the space of monic polynomials 
of degree $n$ with coefficients in $R$, we identify $\mathbb{A}^{m}$ with the product $Q_{e} \times \mathbb{A}_{2 e-1}^{d} \times \mathbb{A}_{e-1}^{c}$. Under this identification, a $k$-rational point of $\mathbb{A}^{m}$ corresponds to a triple

$$
(q(t), \bar{x}(t), \bar{y}(t)) \in\left(t^{e}+k[t]_{<e}\right) \times\left(k[t]_{<2 e}\right)^{d} \times\left(k[t]_{<e}\right)^{c} .
$$

In particular, coordinates in $\mathbb{A}^{m}$ take the form $q^{(n)}, \bar{x}_{i}^{(n)}, \bar{y}_{j}^{(n)}$. Consider the conditions

$$
\begin{aligned}
& p_{1}(\bar{x}(t), \bar{y}(t)) \equiv \cdots \equiv p_{c}(\bar{x}(t), \bar{y}(t)) \equiv 0 \bmod q(t), \\
& \operatorname{det}\left(\frac{\partial\left(p_{1}, \ldots, p_{c}\right)}{\partial\left(y_{1}, \ldots, y_{c}\right)}(\bar{x}(t), \bar{y}(t))\right) \equiv 0 \bmod q(t), \\
& \operatorname{adj}\left(\frac{\partial\left(p_{1}, \ldots, p_{c}\right)}{\partial\left(y_{1}, \ldots, y_{c}\right)}(\bar{x}(t), \bar{y}(t))\right)\left(\begin{array}{c}
p_{1}(\bar{x}(t), \bar{y}(t)) \\
\vdots \\
p_{c}(\bar{x}(t), \bar{y}(t))
\end{array}\right) \equiv\left(\begin{array}{c}
0 \\
\vdots \\
0
\end{array}\right) \bmod q(t)^{2} .
\end{aligned}
$$

Here $\operatorname{adj}(B)$ denotes the classical adjoint of a matrix $B$. As explained in [24] and [10, Sections 3.3 and 3.4], the conditions in formula (10a) are polynomial in the coefficients of $q(t), \bar{x}(t), \bar{y}(t)$, and therefore they define a finite-type subscheme $Z \subset \mathbb{A}^{m}$.

Write the arc $\alpha$ in the coordinates $(x, y)$ of $\mathbb{A}^{N}$ as $\alpha=(a(t), b(t))$, where $a(t) \in k[[t]]^{d}$ and $b(t) \in k[[t]]^{c}$. To $\alpha$ we associate the point $z=\left(t^{e}, \bar{a}(t), \bar{b}(t)\right) \in Z$ given by

$$
\bar{a}(t) \equiv a(t) \bmod t^{2 e}, \quad \bar{b}(t) \equiv b(t) \bmod t^{e} .
$$

It is shown in [24] that $\widehat{Z_{z}}$ gives a (finite-dimensional) formal model for $\alpha$ - that is,

$$
\widehat{X_{\infty, \alpha}} \simeq \widehat{Z_{z}} \hat{\times} \Delta^{\mathbb{N}}
$$

The isomorphism in formula (10c) can be expressed somewhat explicitly in coordinates. We identify $\mathbb{A}_{\infty}^{d}$ with an infinite-dimensional affine space $\mathbb{A}^{\mathbb{N}}$, and we use the notation $\xi(t)$ for points in $\mathbb{A}^{\mathbb{N}}$. Hence coordinates in $\mathbb{A}^{\mathbb{N}}$ take the form $\xi_{i}^{(n)}$, with $1 \leq i \leq d$ and $n \geq 0$. The disk $\Delta^{\mathbb{N}}$ appearing in formula (10c) is the formal neighbourhood of $c(t)$ in $\mathbb{A}^{\mathbb{N}}$, where $c(t):=t^{-2 e}(a(t))_{\geq 2 e}$ is the truncation of $a(t)$ to degrees $\geq 2 e$ divided by $t^{2 e}$. Summarising, we have described coordinates $(x(t), y(t))$ in $\overline{X_{\infty, \alpha}}$ and coordinates $(q(t), \bar{x}(t), \bar{y}(t), \xi(t))$ in $\widehat{Z_{z}} \hat{\times} \Delta^{\mathbb{N}}$. As explained in [24], the isomorphism in formula (10c) gives the relation

$$
x(t)=q(t)^{2} \xi(t)+\bar{x}(t)
$$

and we have

$$
\bar{x}(t) \equiv x(t) \bmod q(t)^{2}, \quad \bar{y}(t) \equiv y(t) \bmod q(t) .
$$

We emphasise that these relations hold only at the level of formal neighbourhoods.

Notice that the point $z \in Z$ depends on the arc $\alpha$, but the scheme $Z$ depends only on the choices of the coordinates $x_{i}, y_{j}$, the equations $p_{l}$ and the order of contact $e$. The choice of coordinates $x_{i}, y_{j}$ also determines the linear projection $\mathbb{A}^{N} \rightarrow \mathbb{A}^{d}$ given by $(x, y) \mapsto x$, and hence the induced map $f: X \rightarrow \mathbb{A}^{d}$.

Definition 10.1. With the foregoing notation, we say that $(Z, z)$ is a Drinfeld model of $X_{\infty}$ at $\alpha$, and that it is compatible with $f$.

We are now ready to state and prove our comparison theorem. 
Theorem 10.2. Let $X \subset \mathbb{A}^{N}$ be an affine scheme of finite type over a perfect field $k$, let $\alpha \in X_{\infty}(k)$ with $\alpha(\eta) \in X_{\mathrm{sm}}$ and let $d:=\operatorname{dim}_{\alpha(\eta)}(X)$. Let $f: X \rightarrow Y:=\mathbb{A}^{d}$ be induced by a general linear projection $\mathbb{A}^{N} \rightarrow \mathbb{A}^{d}$ and let $\beta:=f_{\infty}(\alpha)$. Let $(Z, z)$ be a Drinfeld model compatible with $f$, and let $\widehat{X_{\infty, \alpha}} \simeq \widehat{Z_{z}} \hat{\times} \Delta^{\mathbb{N}}$ be the corresponding DGK decomposition.

1. The composition map

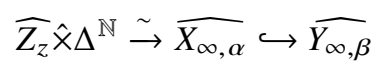

is the completion of a morphism $g: Z \times \mathbb{A}^{\mathbb{N}} \rightarrow Y_{\infty}$.

2. If $X^{0} \subset X$ is the irreducible component of containing $\alpha(\eta)$ and $e:=\operatorname{ord}_{\alpha}\left(\operatorname{Jac}_{X^{0}}\right)$, then the composition map

$$
\widehat{Z_{z}} \hookrightarrow \widehat{Z_{z}} \hat{\times} \Delta^{\mathbb{N}} \stackrel{\sim}{\rightarrow} \widehat{X_{\infty, \alpha}} \hookrightarrow \widehat{Y_{\infty, \beta}} \rightarrow \widehat{Y_{2 e-1, \beta_{2 e-1}}}
$$

is an efficient formal embedding. Moreover, at the level of associated graded rings, we have that

$$
\operatorname{gr}\left(\mathcal{O}_{Z, z}\right)=\operatorname{Im}\left(\operatorname{gr}\left(\mathcal{O}_{Y_{2 e-1}, \beta_{2 e-1}}\right) \rightarrow \operatorname{gr}\left(\mathcal{O}_{X_{\infty}, \alpha}\right)\right)
$$

Proof. We use the notation introduced at the beginning of the section. In particular: we have coordinates $(x, y)$ in $\mathbb{A}^{N}$ such that the projection $\mathbb{A}^{N} \rightarrow Y$ is given by $(x, y) \mapsto x$; we write $\alpha=(a(t), b(t))$, so $\beta=f_{\infty}(\alpha)=a(t)$; we have a space $\mathbb{A}^{m}$ with coordinates $(q(t), \bar{x}(t), \bar{y}(t))$, and $Z \subset \mathbb{A}^{m}$ is defined by the conditions in formula (10a); the point $z \in Z$ is given by $z=\left(t^{e}, \bar{a}(x), \bar{b}(x)\right)$ as in formula (10b); the formal scheme $\widehat{Z_{z}} \hat{\times} \Delta^{\mathbb{N}}$ is contained in the completion of $\mathbb{A}^{m} \times \mathbb{A}^{\mathbb{N}}$ at $(z, c(t))$, where $c(t)=t^{-2 e}(a(t))_{\geq 2 e}$; and the coordinates in this affine space have the form $(q(t), \bar{x}(t), \bar{y}(t), \xi(t))$.

We define a map $\mathbb{A}^{m} \times \mathbb{A}^{\mathbb{N}} \rightarrow Y_{\infty}$ via

$$
(q(t), \bar{x}(t), \bar{y}(t), \xi(t)) \mapsto q(t)^{2} \xi(t)+\bar{x}(t),
$$

and we let $g: Z \times \mathbb{A}^{\mathbb{N}} \rightarrow Y_{\infty}$ be the restriction. It is clear from the discussion surrounding formula (10d)

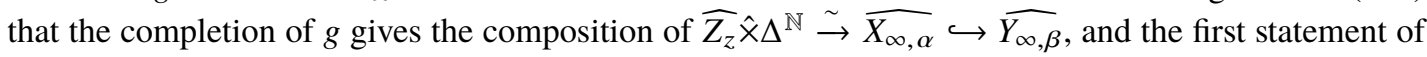
the theorem follows.

We compute the tangent map of $g$ explicitly. With a small abuse of notation where coordinates of elements and coordinate functions are written in the same way, we denote a tangent vector on $Z \times \mathbb{A}^{\mathbb{N}}$ based at a point $(z, c(t))=\left(t^{e}, \bar{a}(t), \bar{b}(t), c(t)\right)$ by

$$
\left(t^{e}+d q(t) \epsilon, \bar{x}(t)+d \bar{x}(t) \epsilon, \bar{y}(t)+d \bar{y}(t) \epsilon, c(t)+d \xi(t) \epsilon\right)
$$

where $d q(t) \in k[t]_{<e}, d \bar{x}(t) \in\left(k[t]_{<2 e}\right)^{d}, d \bar{y}(t) \in\left(k[t]_{<e}\right)^{c}, d \xi(t) \in k[t]^{d}$ and $\epsilon^{2}=0$. The image of such a tangent vector under $g$ is given by

$$
\begin{aligned}
\left(t^{e}+d q(t) \epsilon\right)^{2}(c(t)+ & d \xi(t) \epsilon)+(\bar{x}(t)+d \bar{x}(t) \epsilon) \\
& =\left(t^{2 e} c(t)+\bar{x}(t)\right)+\left(d \bar{x}(t)+d \xi(t) t^{2 e}+2 c(t) d q(t) t^{e}\right) \epsilon \\
& =a(t)+\left(d \bar{x}(t)+d \xi(t) t^{2 e}+2 c(t) d q(t) t^{e}\right) \epsilon .
\end{aligned}
$$

In other words, the tangent map of $g$ at $(z, c(t))$ is given by

$$
d x(t)=d \bar{x}(t)+d \xi(t) t^{2 e}+2 c(t) d q(t) t^{e},
$$


or, in coordinates - recall that $c(t)=t^{-2 e}(a(t))_{\geq 2 e}-$ by

$$
d x_{i}^{(n)}= \begin{cases}d \bar{x}_{i}^{(n)} & \text { if } n<e \\ d \bar{x}_{i}^{(n)}+2 \sum_{k+l=n-e} a_{i}^{(k+2 e)} d q^{(l)} & \text { if } e \leq n<2 e, \\ d \xi_{i}^{(n-2 e)}+2 \sum_{k+l=n-e} a_{i}^{(k+2 e)} d q^{(l)} & \text { if } n \geq 2 e\end{cases}
$$

From this we see that $g$ induces a surjective map between associated graded rings

$$
\varphi: k\left[d x_{i}^{(n)} \mid \underset{1 \leq i \leq d}{n \in \mathbb{N}}\right] \simeq \operatorname{gr}\left(\mathcal{O}_{Y_{\infty}, \beta}\right) \longrightarrow \operatorname{gr}\left(\mathcal{O}_{Z, z}\right) \otimes_{k} k\left[d \xi_{i}^{(m)} \mid \underset{1 \leq i \leq d}{m \in \mathbb{N}}\right] .
$$

We have a commutative diagram

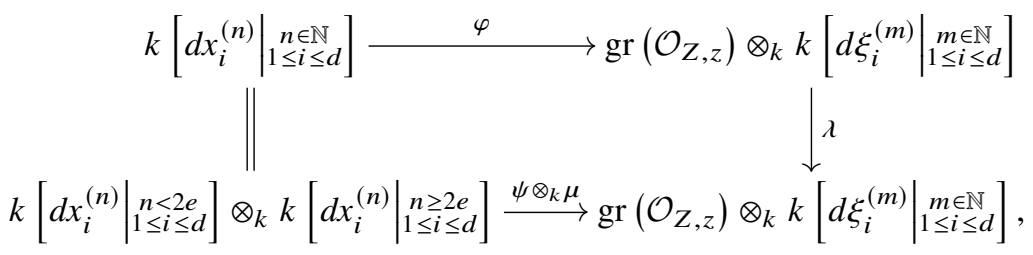

where $\lambda$ is the $\operatorname{gr}\left(\mathcal{O}_{Z, z}\right)$-linear map given by

$$
d \xi_{i}^{(m)} \mapsto d \xi_{i}^{(m)}-2 \sum_{k+l=m-e} a_{i}^{(k+2 e)} d q^{(l)},
$$

$\mu$ is given by $d x_{i}^{(n)} \mapsto d \xi_{i}^{(n-2 e)}$ for $n \geq 2 e$ and $\psi$ agrees with the map $\operatorname{gr}\left(\mathcal{O}_{Y_{2 e-1}, \beta_{2 e-1}}\right) \rightarrow \operatorname{gr}\left(\mathcal{O}_{Z, z}\right)$ induced by the composition

$$
Z \rightarrow Z \times\{c(t)\} \hookrightarrow Z \times \mathbb{A}^{\mathbb{N}} \rightarrow Y_{\infty} \rightarrow Y_{2 e-1},
$$

which is given by

$$
(\bar{x}(t), \bar{y}(t), q(t)) \mapsto \bar{x}(t)+t^{-2 e}(a(t))_{\geq 2 e} q(t)^{2} \bmod t^{2 e}
$$

The map $\lambda$ is invertible, and by Theorem 8.1, the map $\varphi$ is surjective, and thus $\psi$ is surjective as well. This implies that

$$
\operatorname{gr}\left(\mathcal{O}_{Z, z}\right)=\operatorname{Im}\left(\operatorname{gr}\left(\mathcal{O}_{Y_{2 e-1}, \beta_{2 e-1}}\right) \rightarrow \operatorname{gr}\left(\mathcal{O}_{X_{\infty}, \alpha}\right)\right)
$$

and hence the last assertion follows. For the first part of (2), the fact that $\psi$ is surjective implies that the map induced on completions $\widehat{O_{Y_{2 e-1}, \beta_{2 e-1}}} \rightarrow \widehat{\mathcal{O}_{Z, z}}$ is surjective as well. The fact that this is an efficient embedding follows from the injectivity of the corresponding tangent map.

\section{Applications to Mather-Jacobian discrepancies}

Throughout this section, let $X$ be a variety over a field $k$ of characteristic 0 .

Given a prime divisor $E$ on a normal birational model $f: Y \rightarrow X$, we define the Mather discrepancy $\widehat{k}_{E}:=\operatorname{ord}_{E}\left(\operatorname{Jac}_{f}\right)$ and the Mather-Jacobian discrepancy (or simply Jacobian discrepancy) $k_{E}^{\mathrm{MJ}}:=$ $\widehat{k}_{E}-\operatorname{ord}_{E}\left(\operatorname{Jac}_{X}\right)$ of $E$ over $X$. Note that these definitions depend only on the valuation ord $E$ defined by $E$ and not by the particular model chosen. The definition extends to any divisorial valuation $v=q \operatorname{ord}_{E}$, where $q$ is a positive integer, by setting $\widehat{k}_{v}:=q \widehat{k}_{E}$ and $k_{v}^{\mathrm{MJ}}:=q k_{E}^{\mathrm{MJ}}$. When $X$ is smooth, both discrepancies agree with the usual discrepancy of $E$ over $X$. We say that $X$ is MJ-terminal if $k_{E}^{\mathrm{MJ}}>0$ whenever $E$ is exceptional over $X$. As proved in $[34,20]$, this condition is equivalent to the condition 
that if $X \subset Y$ is a closed embedding with $Y$ smooth and $c=\operatorname{codim}(X, Y)$, then for any closed subset $T \subsetneq X$ the pair $(Y, c X)$ has minimal $\log$ discrepancy $\operatorname{mld}_{T}(Y, c X)>1$. We refer to $[22,34,20,26]$ for general studies related to these invariants.

The results of this article, together with a theorem from [21], yield a new proof of the following theorem of Mourtada and Reguera:

Theorem 11.1 ([37, Theorem 4.1]). With the foregoing notation, let $\alpha \in X_{\infty}$ be the maximal arc defining a given divisorial valuation $q \operatorname{ord}_{E}$ (that is, such that $\left.\operatorname{ord}_{\alpha}=q \operatorname{ord}_{E}\right)$. Then $\operatorname{dim}\left(\overline{\mathcal{O}_{X_{\infty}, \alpha}}\right) \geq q\left(k_{E}^{\mathrm{MJ}}+1\right)$.

Proof. We have edim $\left(\mathcal{O}_{X_{\infty}, \alpha}\right)=q\left(\widehat{k}_{E}+1\right)$ by [21, Theorem 11.4], and Theorem 8.5 gives us $\operatorname{ecodim}\left(\mathcal{O}_{X_{\infty}, \alpha}\right) \leq \operatorname{ord}_{\alpha}\left(\operatorname{Jac}_{X}\right)$. It follows then by Proposition 7.4 and Corollary 7.5 that

$$
\operatorname{dim}\left(\overline{\mathcal{O}_{X_{\infty}, \alpha}}\right)=\operatorname{edim}\left(\mathcal{O}_{X_{\infty}, \alpha}\right)-\operatorname{ecodim}\left(\mathcal{O}_{X_{\infty}, \alpha}\right) \geq q\left(k_{E}^{\mathrm{MJ}}+1\right) .
$$

Assume now that $X$ is an affine toric variety. To fix notation, let $T$ be an algebraic $k$-torus, $N:=$ $\operatorname{Hom}_{k}\left(\mathbb{G}_{m}, T\right), M:=\operatorname{Hom}_{\mathbb{Z}}(M, \mathbb{Z}), \sigma \subset N_{\mathbb{R}}$ a rational convex cone and $X:=\operatorname{Spec} k\left[\sigma^{\vee} \cap M\right]$. Note that every $v \in \sigma \cap N$ defines a $T$-invariant divisorial valuation on $X$.

In their recent article [13], Bourqui and Sebag study DGK decompositions of $X_{\infty}$ at arcs that are not fully contained in the $T$-invariant divisor of $X$. The focus is on the open set $X_{\infty}^{\circ} \subset X_{\infty}$ consisting of those arcs whose generic point is in $T$. They prove that for any $\alpha \in X_{\infty}^{\circ}$, the local ring $\mathcal{O}_{X_{\infty}, \alpha}$ depends only on the associated valuation $\operatorname{ord}_{\alpha}$, and in particular so does the minimal formal model [13, Corollary 3.3]. In particular, if we set

$$
X_{\infty, v}^{\circ}:=\left\{\alpha \in X_{\infty}^{\circ} \mid \operatorname{ord}_{\alpha}=v\right\},
$$

then we can denote by $\mathcal{Z}_{v}^{\min }$ the minimal formal model of $X_{\infty}$ at any $\operatorname{arc} \alpha \in X_{\infty, v}^{\circ}$.

The next theorem is one of the main results of [13]. A similar, more general property is proved for elements $v$ satisfying a certain property called $\mathcal{P}_{v}$; we refer to the original source for the precise statement.

Theorem 11.2 ([13, Corollary 6.4]). With the foregoing notation, if $v$ is indecomposable in $\sigma \cap N$, then the associated minimal formal model $\mathcal{Z}_{v}^{\min }$ has $\operatorname{dim}\left(\mathcal{Z}_{v}^{\min }\right)=0$ and $\operatorname{edim}\left(\mathcal{Z}_{v}^{\min }\right)=\widehat{k}_{v}$.

Indecomposable elements $v \in \sigma \cap N$ are characterised by the property that their centres on any resolution of singularity of $X$ are irreducible components of codimension 1 of the exceptional locus [13, Theorem 2.7]. In the terminology of the Nash problem, these form a particular class of essential valuations. By combining Theorem 11.2 with our results, we obtain the following corollary:

Corollary 11.3. Let $X=\operatorname{Spec} k\left[\sigma^{\vee} \cap M\right]$ be an affine toric variety.

1. For any indecomposable element $v \in \sigma \cap N$, we have $k_{v}^{\mathrm{MJ}} \leq 0$.

2. If $X$ is singular and $\mathbb{Q}$-factorial, then $X$ is not MJ-terminal.

Proof. Part (1) follows immediately from Theorems 11.2 and 9.4, and (2) follows from (1) and the observation that if $X$ is singular and $\mathbb{Q}$-factorial, then $\sigma \cap N$ necessarily contains an exceptional indecomposable element. This is just because the exceptional locus of any resolution of singularity of a Q-factorial variety always has pure codimension 1 , and the set of essential (toric) valuations is nonempty if $X$ is singular.

Acknowledgments. We wish to thank Herwig Hauser, Mel Hochster, Hiraku Kawanoue, François Loeser, Mircea Mustaţă and Karl Schwede for useful comments and discussions. We are also very grateful to the referee for their careful reading of the paper and their valuable comments and remarks.

The research of the first author was partially supported by the Austrian Science Fund project P31338 and by NWO Vici grant 639.033.514. The research of the second author was partially supported by NSF grants DMS-1700769 and DMS-2001254, and 
by NSF grant DMS-1440140 while in residence at MSRI in Berkeley during the spring 2019 semester. The research of the third author was partially supported by a grant from the Simons Foundation (638459, RD).

Conflicts of interest. None.

\section{References}

[1] M. Artin, 'Algebraic approximation of structures over complete local rings', Publ. Math. Inst. Hautes Études Sci. 36 (1969), 23-58.

[2] M. Asgharzadeh and M. Tousi, 'On the notion of Cohen-Macaulayness for non-Noetherian rings', J. Algebra 322(7) (2009), 2297-2320. doi:10.1016/j.jalgebra.2009.06.017.

[3] M. F. Atiyah and I. G. Macdonald, Introduction to Commutative Algebra (Addison-Wesley, Reading, MA, 1969).

[4] T. Becker, 'Stability and Buchberger criterion for standard bases in power series rings', J. Pure Appl. Algebra 66(3) (1990), 219-227. doi:10.1016/0022-4049(90)90028-G.

[5] B. Bhatt, 'Algebraization and Tannaka duality', Camb. J. Math. 4(4) (2016), 403-461. doi:10.4310/CJM.2016.v4.n4.a1.

[6] J. Bochnak and W. Kucharz, 'Local algebraicity of analytic sets', J. Reine Angew. Math. 352 (1984), 1-14. doi:10.1515/crll.1984.352.1.

[7] N. Bourbaki, Elements of Mathematics: Commutative Algebra (Hermann, Paris, 1972). Translated from the French.

[8] N. Bourbaki, Elements of Mathematics. Algebra, Part I: Chapters 1-3 (Hermann, Paris, 1974). Translated from the French.

[9] D. Bourqui and J. Sebag, 'The Drinfeld-Grinberg-Kazhdan theorem is false for singular arcs', J. Inst. Math. Jussieu 16(4) (2017), 879-885. doi:10.1017/S1474748015000341.

[10] D. Bourqui and J. Sebag, 'The Drinfeld-Grinberg-Kazhdan theorem for formal schemes and singularity theory', Confluentes Math. 9(1) (2017), 29-64. doi:10.5802/cml.35.

[11] D. Bourqui and J. Sebag, 'Smooth arcs on algebraic varieties', J. Singul. 16 (2017), 130-140.

[12] D. Bourqui and J. Sebag, 'Cancellation and regular derivations', J. Algebra Appl. 18(09) (2019), 1-9.

[13] D. Bourqui and J. Sebag, 'Finite formal model of toric singularities', J. Math. Soc. Japan 71(3) (2019), 805-829.

[14] A. Bouthier, 'Cohomologie étale des espaces d'arcs', Preprint, 2020, arXiv:1509.02203v6.

[15] A. Bouthier and D. Kazhdan, 'Faisceaux pervers sur les espaces d'arcs', Preprint, 2017, arXiv:1509.02203v5.

[16] A. Bouthier, B. C. Ngô and Y. Sakellaridis, 'On the formal arc space of a reductive monoid', Amer. J. Math. 138(1) (2016), 81-108. doi:10.1353/ajm.2016.0004.

[17] W. Bruns and J. Herzog, Cohen-Macaulay Rings, Cambridge Studies in Advanced Mathematics vol. 39 (Cambridge University Press, Cambridge, 1993).

[18] C. Chiu, Local Geometry of the Space of Arcs, PhD dissertation, University of Vienna, 2020.

[19] C. Chiu and H. Hauser, 'On the formal neighborhood of degenerate arcs', Preprint, 2016, URL: https://homepage.univie.ac.at/herwig.hauser.

[20] T. de Fernex and R. Docampo, 'Jacobian discrepancies and rational singularities', J. Eur. Math. Soc. (JEMS) 16(1) (2014), 165-199. doi:10.4171/JEMS/430.

[21] T. de Fernex and R. Docampo, 'Differentials on the arc space', Duke Math. J. 169(2) (2020), $353-396$. doi:10.1215/00127094-2019-0043.

[22] T. de Fernex, L. Ein and S. Ishii, 'Divisorial valuations via arcs', 'Publ. Res. Inst. Math. Sci. 44(2) (2008), 425-448. doi:10.2977/prims/1210167333.

[23] J. Denef and F. Loeser, 'Germs of arcs on singular algebraic varieties and motivic integration', Invent. Math. 135(1) (1999), 201-232. doi:10.1007/s002220050284.

[24] V. Drinfeld, 'On the Grinberg-Kazhdan formal arc theorem', Preprint, 2002, arXiv:math/0203263.

[25] V. Drinfeld, 'Grinberg-Kazhdan theorem and Newton groupoids', Preprint, 2018, arXiv:1801.01046.

[26] L. Ein and S. Ishii, 'Singularities with respect to Mather-Jacobian discrepancies', in Commutative Algebra and Noncommutative Algebraic Geometry, Vol. II, Mathematical Science Research Institute Publications vol. 68 (Cambridge University Press, New York, 2015), 125-168.

[27] L. Ein and M. Mustaţă, 'Generically finite morphisms and formal neighborhoods of arcs', Geom. Dedicata 139 (2009), 331-335. doi:10.1007/s10711-008-9320-7.

[28] D. Eisenbud, Commutative Algebra, Graduate Texts in Mathematics vol. 150 (Springer-Verlag, New York, 1995).

[29] R. Gilmer and W. Heinzer, 'Primary ideals with finitely generated radical in a commutative ring', Manuscripta Math. 78(2) (1993), 201-221. doi:10.1007/BF02599309.

[30] S. Glaz, Commutative Coherent Rings, Lecture Notes in Mathematics vol. 1371 (Springer-Verlag, Berlin, 1989).

[31] M. Grinberg and D. Kazhdan, 'Versal deformations of formal arcs', Geom. Funct. Anal. 10(3) (2000), 543-555. doi:10.1007/PL00001628.

[32] A. Grothendieck, 'Éléments de géométrie algébrique, IV: Étude locale des schémas et des morphismes de schémas. I', Publ. Math. Inst. Hautes Études Sci. 20 (1964), 101-355.

[33] H. Hauser and S. Woblistin, 'Arquile varieties - Varieties consisting of power series in a single variable', Forum Math. Sigma 9 (2021), e78. doi:10.1017/fms.2021.73. 
[34] S. Ishii, 'Mather discrepancy and the arc spaces', Ann. Inst. Fourier (Grenoble) 63(1) (2013), 89-111. doi:10.5802/aif.2756.

[35] C. Lech, 'Inequalities related to certain couples of local rings', Acta Math. 112 (1964), 69-89. doi:10.1007/BF02391765.

[36] H. Matsumura, Commutative Ring Theory, second edn, Cambridge Studies in Advanced Mathematics vol. 8 (Cambridge University Press, Cambridge, 1989).

[37] H. Mourtada and A. J. Reguera, 'Mather discrepancy as an embedding dimension in the space of arcs', Publ. Res. Inst. Math. Sci. 54(1) (2018), 105-139. doi:10.4171/PRIMS/54-1-4.

[38] B. C. Ngô, 'Weierstrass preparation theorem and singularities in the space of non-degenerate arcs', Preprint, 2017, arXiv:1706.05926.

[39] J. Nicaise and J. Sebag, 'Greenberg approximation and the geometry of arc spaces', Comm. Algebra 38(11) (2010), 40774096. doi:10.1080/00927870903295398.

[40] A. J. Reguera, 'A curve selection lemma in spaces of arcs and the image of the Nash map', Compos. Math. 142(1) (2006), 119-130. doi:10.1112/S0010437X05001582.

[41] A. J. Reguera, 'Towards the singular locus of the space of arcs', Amer. J. Math. 131(2) (2009), 313-350. doi:10.1353/ajm.0.0046.

[42] A. J. Reguera, 'Coordinates at stable points of the space of arcs', J. Algebra 494 (2018), 40-76. doi:10.1016/j.jalgebra.2017.09.031.

[43] J. Sebag, 'Primitive arcs on curves', Bull. Belg. Math. Soc. Simon Stevin 23(4) (2016), 481-486.

[44] The Stacks project authors, The Stacks Project (2022). URL: http://stacks.math.columbia.edu.

[45] P. Vojta , 'Jets via Hasse-Schmidt derivations', in Diophantine Geometry, CRM Series vol. 4 (Edizioni della Normale, Pisa, Italy, 2007), 335-361.

[46] H. Whitney, 'Local properties of analytic varieties', in Differential and Combinatorial Topology (A Symposium in Honor of Marston Morse) (Princeton University Press, Princeton, NJ, 1965), 205-244. 